\title{
Multichannel Empirical Orthogonal Teleconnection Analysis: A Method for Space-Time Decomposition of Climate Variability $\mathscr{O}$
}

\author{
BENOIT PARMENTIER \\ Sustainability Solutions Initiative, University of Maine, Orono, Maine \\ NeETI NeETI \\ Department of Natural Resources, The Energy and Resources Institute University, New Delhi, India \\ ELSA NICKL \\ Cooperative Institute for Climate and Satellites, North Carolina State University, and NOAA/National Centers \\ for Environmental Information, Asheville, North Carolina \\ MARCo Millones \\ Department of Geography, University of Mary Washington, Fredericksburg, Virginia
}

(Manuscript received 11 February 2016, in final form 9 January 2017)

\begin{abstract}
With the increasing availability of Earth observation datasets, developing methods for the identification of modes of variability is becoming crucial in Earth system science. These modes, also referred as teleconnections, are useful to understand the global climate system and to predict short-term climate and climate variability. For example, the El Niño-Southern Oscillation (ENSO) phenomenon, a teleconnection with global climate impacts, has been associated with major social, economic, and ecological consequences. In this study, a novel procedure called multichannel empirical orthogonal teleconnection (MEOT) analysis is introduced as a simple extension of the logic of empirical orthogonal teleconnections to uncover the temporal evolution of recurrent space-time patterns. A global monthly sea surface temperature dataset (1982-2007 time series) is used to explore the MEOT method and its differences and similarities with the multichannel singular spectrum analysis (MSSA). Both methods are applied with a 13-month embedding dimension to extract spatiotemporal patterns that exhibit clear basis vectors in quadrature. MSSA extracted four quadratures, and MEOT extracted three. Findings show that MEOT quadratures are more easily related to climate events corresponding to ENSO, South Atlantic Ocean dipole, and Atlantic meridional mode. MSSA identified one quadrature related to ENSO and one related to the quasi-biennial oscillation. The two remaining MSSA quadratures are mixtures of different indices rather than one climate event. Thus, results indicate that, since it does not suffer from a biorthogonality constraint, MEOT is effective at extracting modes of variability in climate datasets, suggesting its potential use in climate research.
\end{abstract}

\section{Introduction}

In this study, we introduce a novel procedure to extract modes of variability of climate that correspond to oscillatory patterns coevolving in space and time. We

Supplemental information related to this paper is available at the Journals Online website: http://dx.doi.org/10.1175/ JAMC-D-16-0072.s1.

Corresponding author: Benoit Parmentier, benoit.parmentier@ maine.edu call the new method "multichannel empirical orthogonal teleconnection" (MEOT) and illustrate its application to a sea surface temperature (SST) dataset as an example. We also explore the differences and similarities between the new MEOT method and the more well-known multichannel singular spectrum analysis (MSSA). Earth's climate system is characterized by spatiotemporal patterns of climate variability at various temporal and spatial scales (IPCC 2007), and, within the system's components, the ocean plays a major role in redistributing heat and in influencing climates around the world (Carissimo et al. 1985; IPCC 2007; Wunsch 
2005). The interannual variability of oceans is known to be a major causal mechanism of climate variability because of its thermal capacity and its impact on the atmosphere (IPCC 2007; Wunsch 2005). Evidence from the scientific literature indicates that slowly varying boundary conditions such as SST modulate time-mean area-averaged rainfall over the tropics (Dirmeyer and Shukla 1993; Shukla 1984) and that patterns of variability in SST at interannual-and-longer time scales arise from the interaction between the atmosphere and the ocean (Deser et al. 2010). For instance, El NiñoSouthern Oscillation (ENSO) constitutes a well-known phenomenon illustrative of an ocean-atmosphere coupled oscillation that propagates in the Earth system and influences the weather worldwide (IPCC 2007; Liu and Alexander 2007). ENSO affects the livelihood of human societies around the world by affecting animal population levels (e.g., fish), primary productivity (Potter et al. 2003), and crop yields through influences on fire, drought, rainfall, and streamflow regimes (Chiew and McMahon 2002; Dai et al. 2004; IPCC 2007; Rosenzweig and Hillel 2008; Trenberth et al. 2002). In a similar way, there are other atmospheric and oceanic climate oscillations that affect large areas, such as the Atlantic multidecadal oscillation (AMO; Knight et al. 2005) and the North Atlantic Oscillation (NAO), which are linked to winter weather events in the North Atlantic Ocean (Cook et al. 2002; IPCC 2007), or the Pacific decadal oscillation (PDO), which has been shown to influence fish production levels in the North Pacific Ocean (Mantua et al. 1997). These climate oscillations are often referred to as teleconnections in the sense that they represent preferred recurrent spatiotemporal patterns or modes (components) of variability of the climate system (Chase et al. 2006; IPCC 2007; Nigam and Baxter 2014). The identification of such climate modes benefits the scientific community and numerous national climate agencies as they provide key information to improve the understanding of Earth's climate system, climate reconstructions, and predictions in short-term climate forecasting (Kim and $\mathrm{Wu}$ 1999; Rotstayn et al. 2010; Smith and Reynolds 2005; van den Dool et al. 2000; van den Dool 2007; Zwiers 1993). The increasing availability of large Earth-observation time series coupled with the need to improve identification of these modes has resulted in a large body of research devoted to the development and refinement of new statistical techniques to identify and decompose large spatiotemporal climate datasets (Compagnucci et al. 2001; Jolliffe 2002; Kim and Wu 1999; Mann and Park 1999; Reynolds et al. 2007; van den Dool 2007).

Frequently used methods include empirical orthogonal function (EOF; also known as principal components analysis), singular spectrum analysis (SSA), and their variants such as extended EOF (EEOF) and MSSA (Broomhead and Jones 1989; Broomhead and King 1986; Jolliffe 2002; Plaut and Vautard 1994; Trenberth et al. 2000; van den Dool 2007; Vautard et al. 1999). There is a close association among SSA, EOF, and their respective variants (Elsner and Tsonis 2013; Ghil et al. 2002), with both the SSA and EOF techniques being eigenvalue-based methods that decompose covariance or correlation matrices derived from climate datasets (Jolliffe 2002; Keppenne and Ghil 1992).

The primary difference between SSA and EOF is the manner by which the covariance is calculated and set up in a matrix format (Elsner and Tsonis 2013). In essence, SSA can be understood as an EOF decomposition on a lagged covariance structure derived by applying a specific lag window (known as the embedding dimension) to the original dataset (Lee 2002; Vautard et al. 1992). Thus, EOF and SSA analyses share similar goals, that is, the extraction of linearly independent patterns from large datasets (Schnur et al. 1993), with the distinction that SSA focuses on the extraction of oscillations that exhibit distinct phase changes over time and temporal patterns shifted in time with a high cross correlation at a specific lag (Jolliffe 2002; Keppenne and Ghil 1992). These extracted patterns can then be interpreted in term of the physical and dynamic understanding of the Earth system. In a similar way, MSSA is an extension of SSA that is intended to detect space-time oscillatory patterns by taking both the spatial and temporal correlations into account (Graham et al. 1987a,b; Lau and Chan 1986). As such, MSSA is mathematically equivalent to the EEOF (Compagnucci et al. 2001; Graham et al. 1987b; Latif and Graham 1992; Preisendorfer and Mobley 1988; Weare and Nasstrom 1982) since both techniques are spectral decompositions of block covariance or correlation matrices intended to extract space-time patterns. MSSA can therefore be viewed as a form of EEOF analysis that is conducted on an extended field corresponding to a series of lagged variables (Jolliffe 2002; Wilks 2011). To conduct an MSSA, geophysical fields are effectively concatenated to form an extended dataset from which modes of variability can be extracted. Since the concatenated fields are lags of the same variable, the technique is aimed at finding oscillatory modes in both space and time (Jolliffe 2002). Although the MSSA and EEOF techniques have both been widely used in the climate literature, they suffer from a major drawback in that they inherit the property of biorthogonality, which constrains the extraction of modes and often leads to mixed modes (van den Dool et al. 2000). Such mixed modes are difficult to interpret and may not correspond to existing physical phenomena 
(van den Dool 2007). In consequence, researchers have developed over the years alternative statistical methods to relieve the biorthogonality constraint (Jolliffe 2002). In the EOF literature, rotation (Groth and Ghil 2011; Jolliffe et al. 2002; Mestas-Nuñez 2000; Richman 1986), regionalization (Jolliffe 2002), and empirical orthogonal teleconnection (EOT) analysis (van den Dool et al. 2000; Smoliak and Wallace 2015) are all presented as effective measures to attain these goals. Among them, EOT is considered to be the computationally simplest way to achieve such goals because it is based on multiple linear regression, and EOT results are very closely related to rotated EOF modes (van den Dool 2007). Furthermore, EOFs are designed to provide a concise description of global variance, which may not always be desirable, whereas EOT describes variance over one dimension only, that is, either time or space, and often displays more regionalization (van den Dool 2007).

Since EOT in its current form (van den Dool 2007) is not intended as a method for uncovering oscillatory patterns from spatiotemporal datasets, we introduce in this study a variant of EOT that is aimed at the extraction of modes coevolving in space and time. The novel method, MEOT, is introduced and applied to a global SST dataset to extract oscillatory patterns that coevolve in space and time. In both MEOT and MSSA, oscillatory events are characterized by pairs of modes with similar spatial and temporal patterns but shifted in time and/or space. These pairs of modes are called quadratures because they correspond to the same oscillatory phenomenon but out of phase, that is, shifted by a quadrant, or $90^{\circ}$ (Jolliffe 2002). The central purpose of this paper is to introduce MEOT and to compare it with MSSA so as to assess its ability to extract patterns in quadratures that exhibit phase changes in space and time.

\section{Data}

National Oceanic and Atmospheric Administration (NOAA) Optimum Interpolation Sea Surface Temperature, version 2 (OISSTv2), is used in the analysis. This global SST time series spans 1982-2007 with time samples at a monthly temporal frequency and a spatial resolution of $1^{\circ}$ in latitude and longitude. The dataset corresponds to a stack of 312 images (geophysical fields) with 180 rows and 360 columns, amounting to a total of 64800 pixels per image. The SST dataset (http://www. esrl.noaa.gov/psd/data/gridded/data.noaa.oisst.v2.html) was obtained from the NOAA/Climate Prediction Center (CPC). It results from a fusion of remotely sensed observations from the NOAA AVHRR sensor and in situ measurements from gauges, buoys, and boats (Reynolds et al. 2002).
TABLE 1. Twelve climate indices are utilized to interpret the spatiotemporal modes extracted by MEOT and MSSA.

\begin{tabular}{ll}
\hline \hline Indices & \multicolumn{1}{c}{ Teleconnections: Name and references } \\
\hline PNA & $\begin{array}{l}\text { Pacific North American index (NOAA/CPC) } \\
\text { North Atlantic Oscillation (NOAA/CPC; Jones et al. } \\
\text { 1997) }\end{array}$ \\
TNA & Tropical Northern Atlantic (Enfield et al. 1999) \\
TSA & Tropical Southern Atlantic (Enfield et al. 1999) \\
Niño-3.4 & Niño-3.4 index (NOAA/CPC) \\
PDO & Pacific decadal oscillation (NOAA/CPC; Mantua \\
& et al. 1997) \\
AO & Arctic Oscillation (NOAA/CPC) \\
AAO & Antarctic Oscillation (NOAA/CPC) \\
AMM & Atlantic meridional mode (Chiang and Vimont 2004) \\
AMO & Atlantic multidecadal oscillation (Enfield et al. 2001; \\
& Knight et al. 2005) \\
QBO & Quasi-biennial oscillation (NOAA/CPC) \\
SAOD & South Atlantic Ocean dipole (Nnamchi et al. 2011) \\
\hline
\end{tabular}

In addition, the study utilizes 12 climate teleconnections at a monthly temporal resolution (Table 1) that were obtained from the NOAA/Earth System Research Laboratory (http://www.esrl.noaa.gov/psd/data/ climateindices/list/). These correspond to some of the major modes of variability documented in the climate literature and are used in the interpretation of modes extracted from the MEOT analyses.

\section{Methods}

\section{a. MEOT}

The MEOT is a technique that extends the EOT analysis by working on lagged datasets. The origin of the EOT method may be traced back to the systematic search for teleconnections (van den Dool et al. 2000) that was conducted in the past by correlating every base point to all of the other points (Namias 1981) and by identifying the most important base points using some diagnostic criterion such as teleconnectivity (Wallace and Gutzler 1981). The EOT method (van den Dool et al. 2000) is a regression-based technique that provides a refinement to the selection problem by identifying in succession the base points that explain the most variance at all points including the base point from the datasets. The first base point, named EOT1, corresponds to the base point that maximizes the varianceweighted sum of the coefficient of determination $R^{2}$. Subsequent EOTs are found by applying the same criterion but removing the effect of the previous EOTs by using the regression residuals. By repeating this mathematical procedure, researchers define a set of base points that are orthogonal in the temporal dimension (van den Dool 2007). A spatial EOT pattern is obtained 
by projecting the EOT basis function on the temporal dimension of grid locations, creating an EOT-loading image for each mode. By design, an EOT basis function is orthogonal in a unique dimension and does not suffer from the biorthogonality constraint, thus enhancing interpretability (van den Dool et al. 2000). In the context of this research, we extended the EOT method to study modes of variability from an extended lagged field-hence its name "multichannel EOT." We use the word multichannel to inform the reader that the EOT is carried on a lagged dataset in a manner similar to EOF being performed on a lagged dataset for MSSA.

The method may be summarized in two main steps: lagging and EOT. The first step, lagging, involves the conversion of the univariate (SST) time series dataset into a multivariate dataset by temporally lagging the original SST data. The second step involves an EOT analysis on this extended multivariate dataset.

\section{b. $M S S A$}

The MSSA method is an eigenvalue-based decomposition technique that is conducted on the correlation or covariance matrix of a lagged space-time dataset. In MSSA, the use of lag variables results in special forms of covariance or correlation matrices that approximate block Toeplitz matrices when time series are stationary in the lag window (Elsner and Tsonis 2013; Jolliffe 2002). Toeplitz matrices display basis functions that are in quadrature in a manner similar to sine and cosine functions (Elsner and Tsonis 2013; Watkins and Meyer 2001). The MSSA basis functions - that is, the MSSA modes-do not, however, correspond to analytical functions (sines and cosines) but are extracted empirically from the lagged dataset by the eigen-based decomposition (Jolliffe 2002). Similar to other eigenvalue-based techniques, MSSA possesses the property of biorthogonality, which means that mode scores and loadings are orthogonal.

\section{c. Data matrix, biorthogonality, and spectral decomposition}

The SST lagged dataset used in this research can be conceptually thought of as a cube whose dimensions correspond respectively to time, space, and phase in a manner similar to the representation proposed by Berry (1964) and Ghil et al. 2002. Analyses are carried out using the S-mode orientation, where $n_{s}$ refers to the number of grid points of the geophysical field (64800 grid points of SST), $n_{t}$ refers to the number of monthly lagged observations represented in maps (300 maps as result of 312 months with 13 complete lags), and
$L$ refers to the size of the lag window of the phase dimension (13).

To conduct the spectral decomposition, the data cube must be reduced to a two-dimensional block matrix $\mathbf{X}$ from which the covariance or correlation $\mathbf{R}$ and $\mathbf{R}^{a}$ matrices can be obtained (Fig. S1 in the online supplementary material; this and subsequent supplemental figures are denoted by the leading " $S$ "). We derive matrix $\mathbf{X}$ by concatenating the lag fields together and expanding the spatial dimension to $n^{\prime}=L \times n_{s}$, that is, $13 \times 64800$ variables standardized in time (note that only land pixels are considered in the analysis and therefore the effective number is lower). Thus, the resulting lagged data matrix $\mathbf{X}$ has dimension $n^{\prime} \times n_{t}$, with rows containing grid points and columns corresponding to lag times. In the climate literature, however, the most widely used setup utilizes the transpose of $\mathbf{X}$, that is, $\mathbf{X}^{\mathrm{T}}$. Under this matrix setup, the variables are the spatial locations, or grid points (columns), and the temporal dimension is the set of observations (rows). This type of setup leads to S-mode MSSA and MEOT analyses (Jolliffe 2002). From $\mathbf{X}^{\mathrm{T}}$, two symmetric matrices can be derived: a matrix $\mathbf{R}$ that represents the relationship between the $n^{\prime}$ spatial grid locations over an embedded lag dimension and a matrix $\mathbf{R}^{a}$ that represents the relationship between $n_{t}$ lagged maps (Fig. S1). In MSSA, both the $\mathbf{R}$ and $\mathbf{R}^{a}$ matrices are diagonalized, and their eigenvalues are equal because the singular value decompositions (SVD) of data matrices $\mathbf{X}$ and $\mathbf{X}^{\mathrm{T}}$ are the same; their identical eigenvectors are recoverable by permuting and rescaling the left and right SVD eigenvectors (Jolliffe 2002; Watkins and Meyer 2001; Wilks 2011). Hence, MSSA is a biorthogonal decomposition that decomposes both the matrix $\mathbf{R}$ and $\mathbf{R}^{a}$, in contrast to MEOT, which diagonalizes either the matrix $\mathbf{R}$ or $\mathbf{R}^{a}$ (van den Dool 2007). Because MEOT is based on regression, the researcher must select the direction of the dimension to be orthogonal. In the S-mode orientation, the time dimension is chosen to be orthogonal, which leads to the diagonalization of $\mathbf{R}$.

\section{d. Method workflow}

The original SST dataset is composed of a monthly time series of 312 images spanning a 26-yr time period. MEOT and MSSA were carried out on the SST dataset through a series of four main steps. The first step consists of calculating temporal anomalies by removing the longterm monthly mean from the corresponding calendar months (Fig. 1) to concentrate the analysis on the interannual variability, following the practice of the climate literature (van den Dool 2007).

The second step consists of lagging the dataset to create a series of 13 fields corresponding to a lag window 


\section{ANALYSIS WORKFLOW}

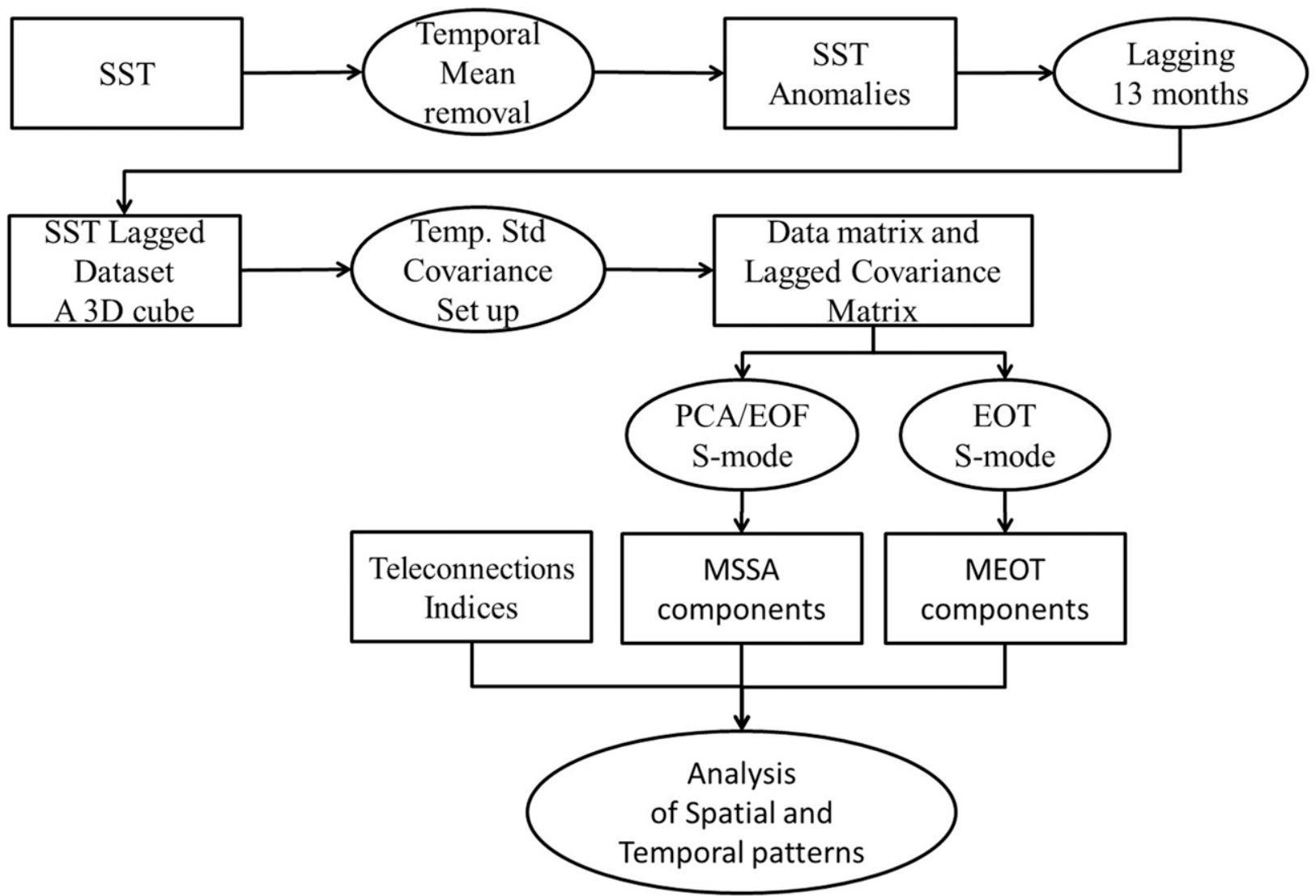

FIG. 1. Methodological workflow for the MEOT analyses: 1) Data anomalies are standardized and lagged in a 13-month window; 2) a lagged correlation matrix is produced and decomposed using the EOT in S mode corresponding respectively to MEOT analyses (see text for details).

$L$ (embedding dimension) that is equivalent to 13 months in a manner similar to Ghil et al. (2002). This lagging procedure reduces the extent of the time series length to $25 \mathrm{yr}$ (the length of the time series is cut by 12 months). In consequence, after the second step, each spatial field (or image) is composed of $1^{\circ}$ grid points (or pixels) with time series of 300 months (equivalent to $25 \mathrm{yr}$ ).

The third step consists of standardizing each grid point using the mean and standard deviation in time. In consequence, the weight of each grid point is solely dependent on its temporal standard deviation and its area (cosine of the latitude). Under this setup, the total space-time variance derived from the dataset can be recovered from two covariance-correlation matrices $\mathbf{R}$ and $\mathbf{R}^{a}$ (van den Dool 2007). In this research, we use the $\mathbf{R}$ matrix, which corresponds to an S-mode analysis as described in section 3c.

The fourth step consists in applying EOT and EOF decompositions on the lagged dataset to extract the
MEOT and MSSA modes of variability (Fig. 1). For MEOT, this is done using the EOT algorithm by computing the correlation between each base point of the lagged datasets (hence the name MEOT). The base point that explains the most variance is the first MEOT. Its temporal profile is correlated back (or projected) to obtain the spatial patterns of MEOT modes of variability. Because the dataset has been lagged as described before, the length of the temporal profiles are cut by 12 while the spatial patterns have been expanded to represent the 13-map sequence. For MSSA, we apply the EOF-based algorithm to the covariancecorrelation matrix from the lag dataset to extract MSSA modes. We obtain maps corresponding to loadings and temporal profiles corresponding to scores. MSSA and MEOT modes are interpreted using the NOAA teleconnections indices using cross-correlation functions. In addition to temporal profiles (bases), the two-dimensional spatial-loading maps also provide 
TABLE 2. Absolute extremum value for lag cross-correlation functions between MEOT modes (lag in parentheses).

\begin{tabular}{lcclcccccc}
\hline \hline & MEOT1 & MEOT3 & MEOT4 & MEOT7 & MEOT8 & MEOT9 & MEOT10 & MEOT15 & MEOT16 \\
\hline MEOT1 & $1(0)$ & $0.72(6)$ & $0.67(-6)$ & $-0.06(7)$ & $0.35(-13)$ & $-0.13(-13)$ & $0.17(-13)$ & $0.08(-3)$ & $-0.13(13)$ \\
MEOT3 & $0.72(-6)$ & $1(0)$ & $0.71(-12)$ & $0.12(-7)$ & $-0.24(-8)$ & $0.11(-8)$ & $-0.13(4)$ & $0.09(-9)$ & $-0.14(9)$ \\
MEOT4 & $0.67(6)$ & $0.71(12)$ & $1(0)$ & $0.15(6)$ & $-0.34(5)$ & $-0.3(-13)$ & $-0.15(2)$ & $-0.13(-4)$ & $-0.16(-5)$ \\
MEOT7 & $-0.06(-7)$ & $0.12(7)$ & $0.15(-6)$ & $1(0)$ & $-0.25(13)$ & $-0.22(8)$ & $0.14(-6)$ & $0.18(13)$ & $0.77(5)$ \\
MEOT10 & $0.17(13)$ & $-0.13(-4)$ & $-0.15(-2)$ & $0.14(6)$ & $0.17(11)$ & $-0.19(12)$ & $1(0)$ & $0.91(-6)$ & $0.18(13)$ \\
MEOT15 & $0.08(3)$ & $0.09(9)$ & $-0.13(4)$ & $0.18(-13)$ & $0.13(13)$ & $-0.09(5)$ & $0.91(6)$ & $1(0)$ & $0.17(-7)$ \\
MEOT16 & $-0.13(-13)$ & $-0.14(-9)$ & $-0.16(5)$ & $0.77(-5)$ & $0.17(-13)$ & $-0.11(10)$ & $0.18(-13)$ & $0.17(7)$ & $1(0)$ \\
\hline
\end{tabular}

visual cues for the identification of teleconnection patterns.

\section{Results}

One of the unique properties of MEOT and MSSA is their ability to uncover moving events by extracting modes that illustrate the development of phenomena in different phases (Moron et al. 1998). Determining moving modes may be done by searching for "quadratures" that correspond to cross-correlation functions exhibiting clear peaks. Modes in quadrature correspond to a pair of temporal basis functions with similar temporal patterns shifted in time and a pair of map sequences with similar spatial patterns shifted in time (Jolliffe 2002; Wang 2001). The literature does not provide a clear threshold value to identify mixing or nonmixing in modes, but Kim and Wu (1999) suggest no mixing if the leading lag correlation is 0.9 and all others are below 0.1 . We consider this rule to be arbitrary, however, and therefore we report the extrema (absolute maximum/minimum) of all cross-correlation values within the length of the embedding dimension between lag -13 and lag +13 (Tables 2 and 3 ). Following this rule, modes are defined to be in quadrature if they have an absolute-value cross-correlation extremum of at least $0.6 \mathrm{and} /$ or have spatial and temporal patterns related to known teleconnections. Using such rules, we found three quadratures for the MEOT analysis and four quadratures for MSSA.

\section{a. MEOT1, MEOT3, and MEOT4: ENSO}

The first and third MEOT modes exhibit spatial and temporal patterns that are related to ENSO. The two map sequences portray overlapping phases of a prototypical ENSO event (Fig. 2), with MEOT1 related to El Niño phase. El Niño events are characterized by the displacement of warm waters from the western Pacific Ocean to the eastern Pacific following a weakening or reversal of the easterly trade winds over the equatorial Pacific. MEOT1 shows the anomaly and timing associated with lag 7 in the lag window and is consistent with an ENSO event (Fig. 2, top). MEOT3, however, is temporally offset and shows the lead-up whereby colder waters in the eastern Pacific are replaced by warmer waters; this pattern would be associated with Kelvin waves moving warm water eastward and taking about 3 months to cross the Pacific (Fig. 2, bottom). The two sequences are 6 months in quadrature as apparent from the maximum of the cross-correlation function (correlation coefficient $r=0.2$ ) between the MEOT temporal profiles (Fig. 3). The lag cross correlation of MEOT profiles peaks at 6 , which suggests a lag of 6 months, similar to the one observed in the map sequences. Comparison of the temporal profiles with teleconnection indices confirms that pair MEOT1-MEOT3 relates the most to the ENSO event (Table 4) as evidenced by correlations with the Niño-3.4 index of 0.82 and 0.73 for MEOT1 and MEOT3, respectively. Results indicate that all other climate indices have correlations that are below the 0.5 threshold (Table 4 and Fig. S5). Thus, findings from the lag-correlation analyses and the spatial patterns indicate that the pair MEOT1-MEOT3 relates to the ENSO event at different lags in time and provides a spatiotemporal representation of a prototypical development of an El Niño event. We found that MEOT4 also displays a spatial pattern related to ENSO and has a correlation of 0.63 with the Niño-3.4 index (Table 4). The spatial sequence from MEOT4 follows by 6 months the spatial sequence of MEOT1. From the cross correlation and spatial pattern (see Fig. S6), we postulate that this component corresponds to the end of the sequence of an ENSO event. In terms of index, MEOT1 and MEOT3 would be more useful for prediction and monitoring because they provide a clearer representation of the ENSO phenomenon at early and full stages.

\section{b. MEOT7 and MEOT16: AMM}

The pair MEOT7-MEOT16 exhibit spatial patterns (Fig. 4) related to the Atlantic meridional mode (AMM) characterized by contrasting warm and cold anomalies in the Canary Current and the Sargasso Sea, respectively. This mode is characterized by an anomalous 


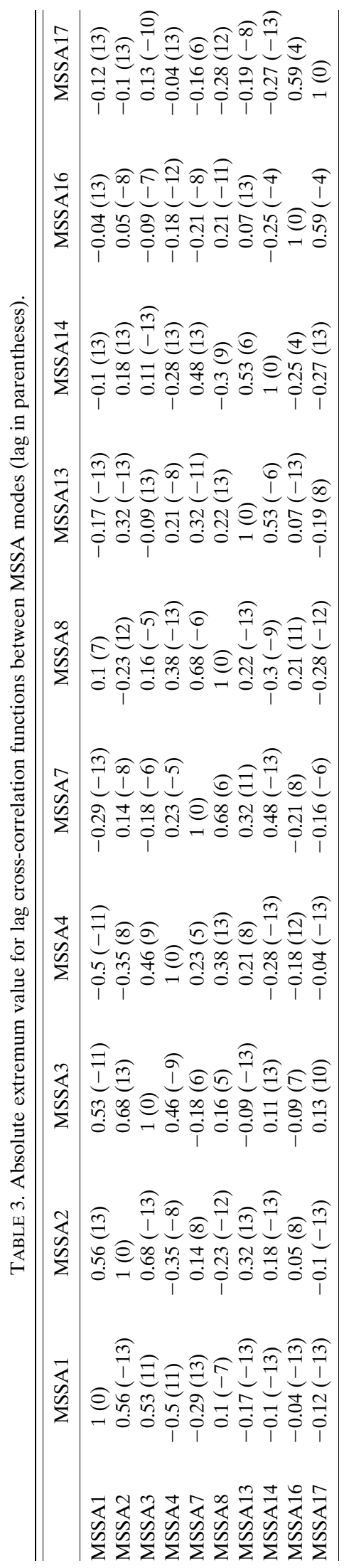

SST gradient over the intertropical convergence zone (ITCZ; Chiang and Vimont 2004) with a warm pool of water clearly visible on most of the map sequences (Fig. 4). In the literature, this anomalous warm gradient has been linked to winds and convection influencing sea level pressure and producing a displacement of the ITCZ toward the warmer hemisphere (Chiang and Vimont 2004; Kossin and Vimont 2007). Chiang and Vimont (2004) report that variations in the trade winds in the subtropical Northern Hemisphere drive the AMM pattern. The AMM buildup is apparent from lag 9 in MEOT16 (Fig. 4, bottom) and continues in MEOT7 with warm pools of water strengthening until lag 7 (Fig. 4, top). The lag cross correlation between MEOT16 and MEOT7 temporal profiles confirms that the two modes are in quadrature, with a peak of correlation of 0.77 occurring at lag 5 , which suggests that MEOT16 leads MEOT7 by 5 months (Fig. 5 , bottom). Temporal profiles for MEOT7 and MEOT16 display a strong relationship with AMM, with correlations of 0.63 and 0.53 , respectively (Table 4). Table 4 reveals, however, that both MEOT7 and MEOT16 also display a strong correlation with the tropical North Atlantic (TNA) climate index, with correlation values of 0.61 and 0.56 , respectively (see the online supplementary material for a visualization using bar plots in Fig. S7). This may arise from the fact that both the AMM and the TNA index, which has not been identified as a distinct teleconnection, overlap in the same spatial domain (Enfield et al. 1999).

\section{c. MEOT10 and MEOT15: SAOD}

The pair MEOT10-MEOT15 exhibits a spatial pattern characteristic of the South Atlantic Ocean dipole (SAOD), with warm anomalies in the Gulf of Guinea and cold anomalies in the South Atlantic Gyre (Fig. 6). The SAOD teleconnection influences weather patterns in equatorial Africa and has been linked to precipitation patterns in West Africa (Nnamchi and Li 2011; Nnamchi et al. 2011). Under SAOD conditions, the disappearance of trade winds leads to the appearance of westerly winds and the buildup of a warm pool of water in the eastern part of the tropical South Atlantic Ocean where otherwise cold waters from the Benguela Current dominate. Similarities with the El Niño event have led many authors to link the SAOD to the Atlantic Niño teleconnection (Florenchie et al. 2003), but work from Nnamchi et al. (2011) indicates that the SAOD is an independent mode of variability. The pair of map sequences depicts a prototypical SAOD event as evidenced by the gradual transition from cold to warm waters in the eastern part of the tropical South Atlantic and the building up of a cold pool in the South Atlantic Gyre. As expected, 


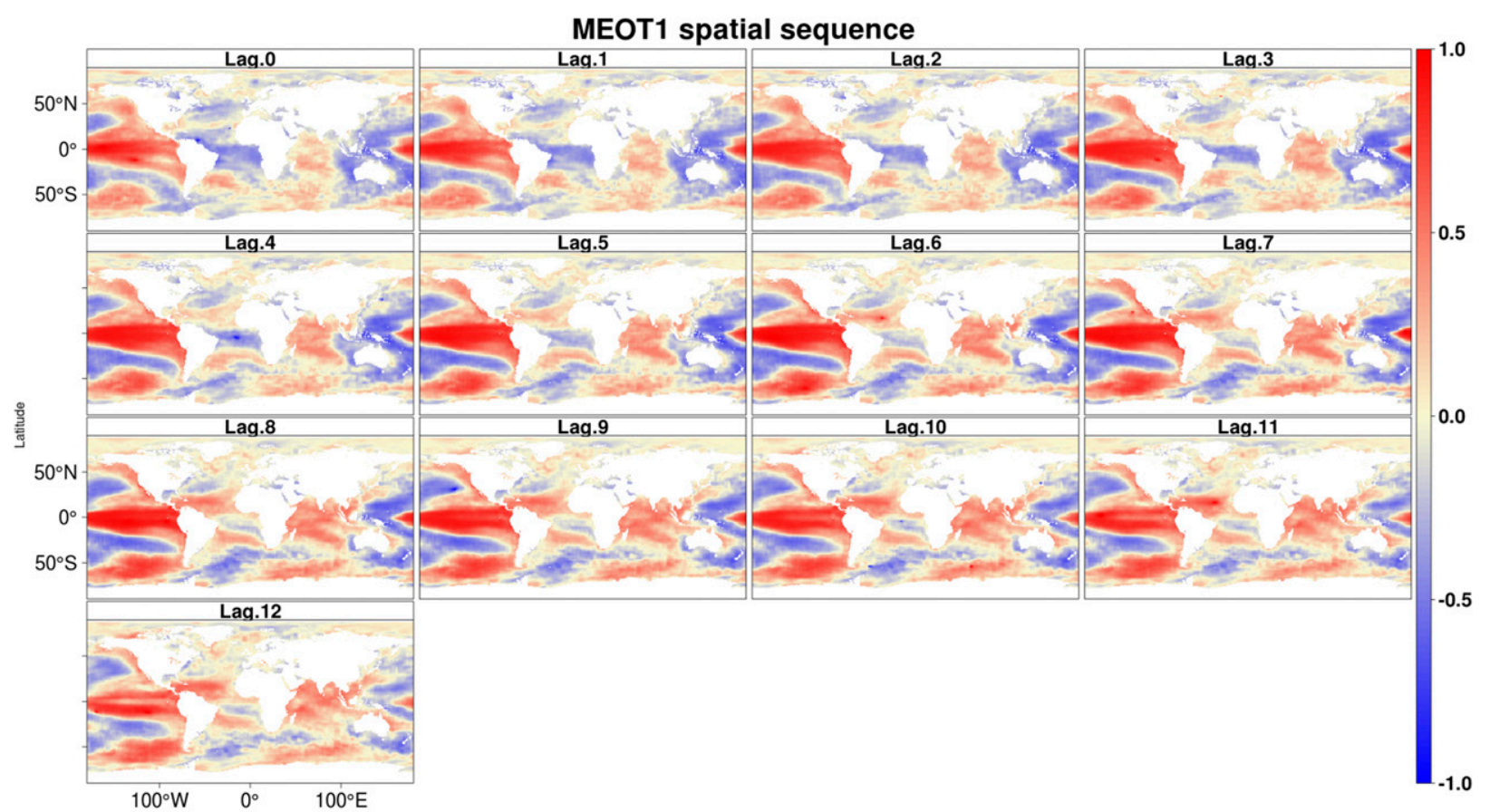

MEOT1 spatial sequence

MEOT3 spatial sequence

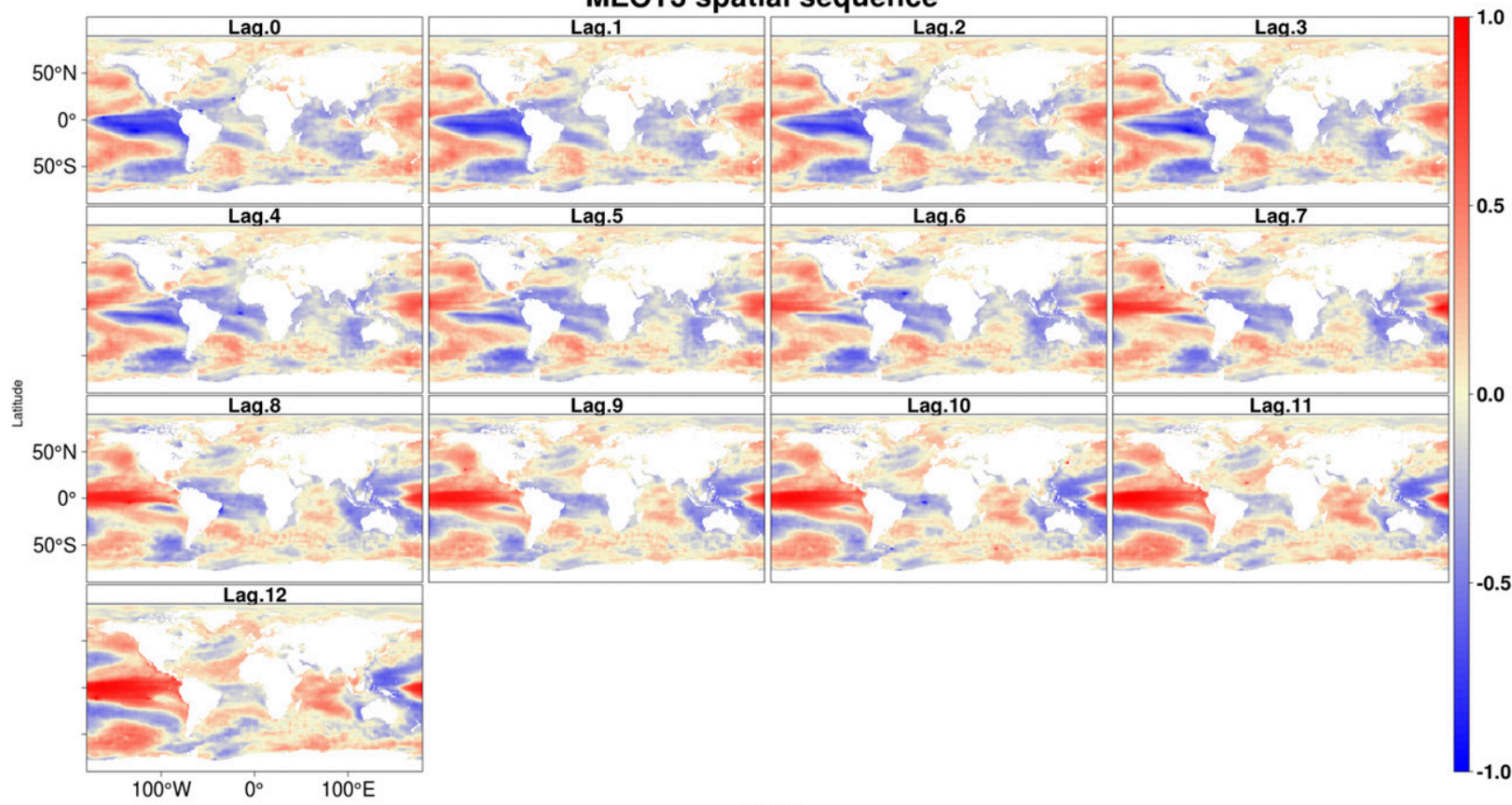

Longitude

FIG. 2. Temporal map sequences for (top) MEOT1 and (bottom) MEOT3 illustrate the unfolding of a typical ENSO event in series of 13 successive phases. MEOT3 shows the buildup to El Niño, and MEOT1 shows strengthening of El Niño conditions.

spatial patterns are shifted and the timing of the transition differs, with the gradual warming peaking at lag 10 (in red) in MEOT10 and at lag 4 in MEOT15 (Fig. 6).

The cross-correlation lag analysis gives further credence to the interpretation of the quadrature as being
SAOD; the pair of temporal profiles displays strong correlations with the SAOD index (Table 4), with values 0.62 and 0.61 for MEOT10 and MEOT15, respectively (Fig. 7). We also found that the MEOT10-MEOT15 pair shows strong correlations with the tropical South 


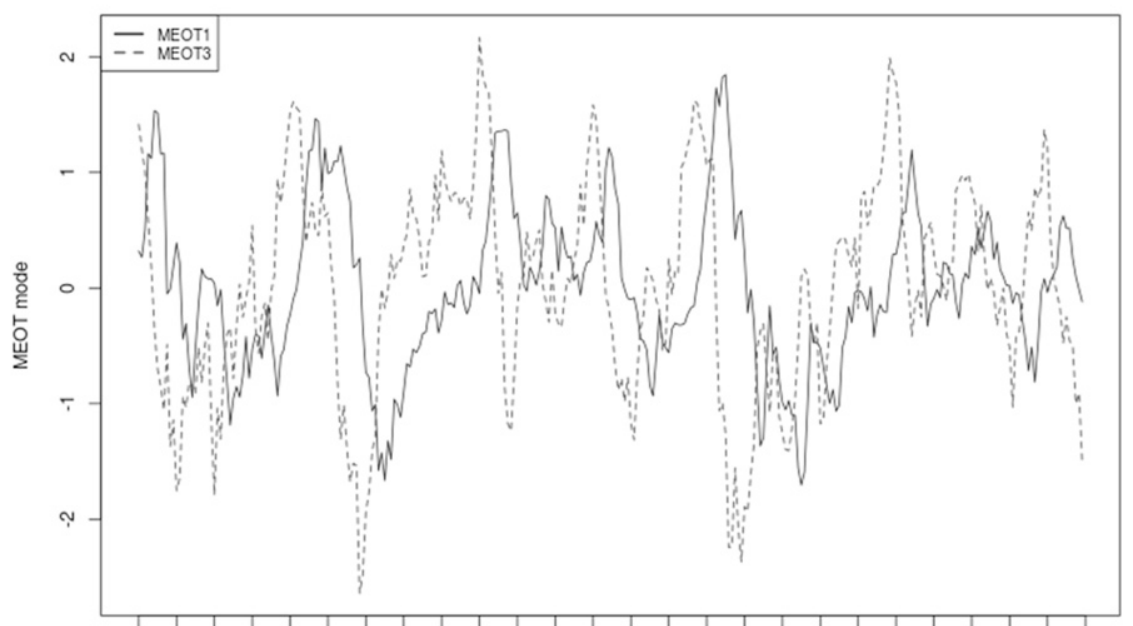

$\begin{array}{llllllllllllllllllllllllll}82 & 83 & 84 & 85 & 86 & 87 & 88 & 89 & 90 & 91 & 92 & 93 & 94 & 95 & 96 & 97 & 98 & 99 & 00 & 01 & 02 & 03 & 04 & 05 & 06 & 07\end{array}$

Time (month)

MEOT1 and MEOT3 lag analysis

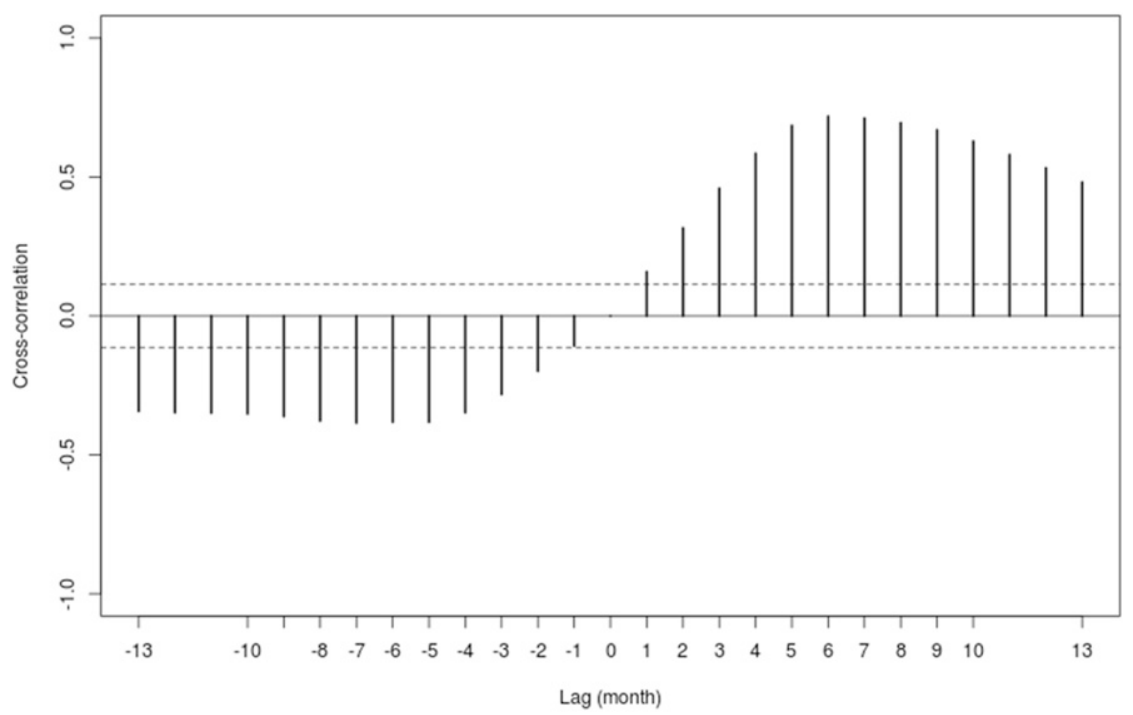

FIG. 3. (top) The temporal profiles for MEOT1 (solid) and MEOT3 (dashed) show the similarities in their temporal patterns. (bottom) The lag cross-correlation function indicates that the modes are in quadrature with a peak of cross correlation of 0.72 at lag 6 . The dashed lines in the bottom graph represent the $95 \%$ confidence interval.

Atlantic (TSA) climate index, with values of 0.64 and 0.59 , respectively (Table 4; see the online supplementary material for a visual representation in Fig. S8). This is not surprising since the TSA index is measured in a similar spatial domain. We interpret this mode as SAOD because the pattern uncovered by MEOT10 and MEOT15 more clearly relates to the dipole from the SAOD phenomenon described in Nnamchi et al. (2011). The quadrature is also confirmed by the lag crosscorrelation analysis, which displays a very high peak of
0.91 at lag -6 , suggesting that MEOT10 precedes MEOT15 by 6 months.

\section{d. MSSA}

We analyzed 20 MSSA modes and found four quadratures: MSSA1-MSSA2-MSSA3-MSSA4, MSSA7MSSA8, MSSA13-MSSA14, and MSSA16-MSSA17. We performed a cross-correlation analysis with a lag window of 13 and provide the extremum values with the corresponding lags for the identified quadratures (see 


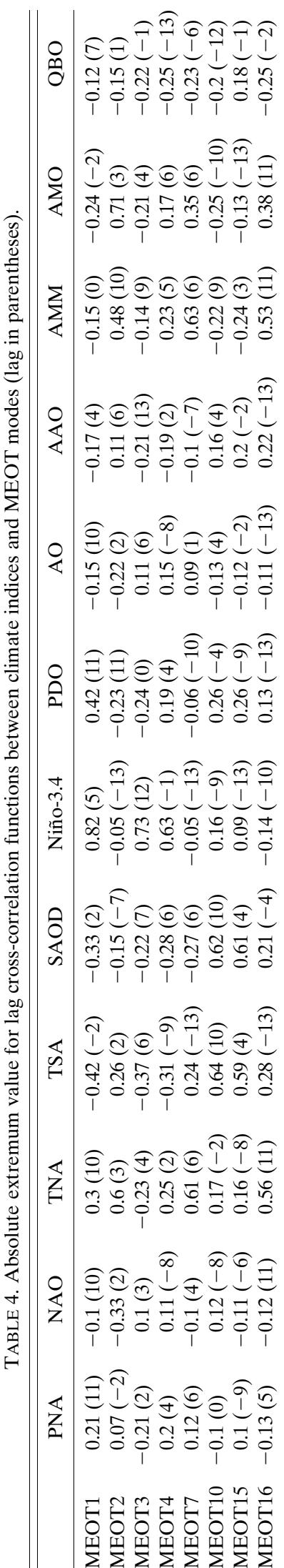

Fig. S3 for a table with the full 20 MSSA). Correlations with teleconnection indices are found in Table 5 and illustrate the multiple correlations for many MSSA, suggesting some mixing of modes (see Fig. S9 for cross correlations with 20 MSSA modes). Although there are four MSSA components with cross correlation above 0.5 for the first quadratures, we matched MSSA1 and MSSA3 as the most plausible quadrature using the map patterns (Fig. S10) and the cross correlation of -0.57 with Niño-3.4 (Table 5). Thus, we identified that quadratures MSSA1-MSSA3 and MSSA13-MSSA14, which display correlations above 0.5 , are related to the ENSO and quasi-biennial oscillation (QBO) teleconnection indices, respectively. The most dominant source of variability is an ENSO event that appears prominently in MSSA1, MSSA2, MSSA3, and MSSA4 (the MSSA spatial patterns visible in Figs. S10 and S13). MSSA6 displays a correlation value of -0.56 at lag 8 with TSA (Fig. S9). MSSA7 and MSSA 8 are in quadrature (cross correlation of 0.68; Table 3 and Fig. S15), but we do not have a definite labeling because the correlations are lower than 0.5 (Table 5 and Fig. S9). The highest correlations are -0.45 and -0.45 with SAOD teleconnections (Table 5 and Fig. S16), and the spatial pattern also suggests some connection with SAOD movements in the Atlantic Ocean (Fig. S14). We find that MSSA13-MSSA14 displays correlations with the QBO index of -0.46 and -0.52 for MSSA13 and MSSA14, respectively (Table 5 and Fig. S19), with a cross-correlation peak of 0.54 (Table 3; see also Fig. S18). According to Huang et al. (2012), strong SSTanomaly patterns exist only in certain QBO phases, and they found significant negative anomalies over the eastern and central tropical Pacific in phase 3, moving westward in phase 4 , and warm temperature anomalies over the western Pacific Ocean. This pattern can be found in lags 10,11,12, and 13 of MSSA13, and lesssignificant negative anomalies are also found in lags 610 of MSSA14 (Fig. S17). There are other significant anomalies in the tropical Atlantic and Indian Oceans that may reflect the teleconnection of the tropical Pacific with the Atlantic (Huang et al. 2012).

We found that the pair MSSA16 and MSSA17 has a cross correlation that is close to the 0.6 threshold ( 0.59 in Table 5 and Fig. S21), but the components do not display strong correlations with any indices (Table 5; see the visualization in Fig. S22 of the online supplementary material); all cross correlations are lower than 0.3. The MSSA16 and MSSA17 spatial pattern is also inconclusive (Fig. S20).

The results suggest that the MSSA1-MSSA3 pair is in quadrature and displays a strong relationship with the ENSO event but with some mixing with other climate modes. The lag analysis indicates that the MSSA1 

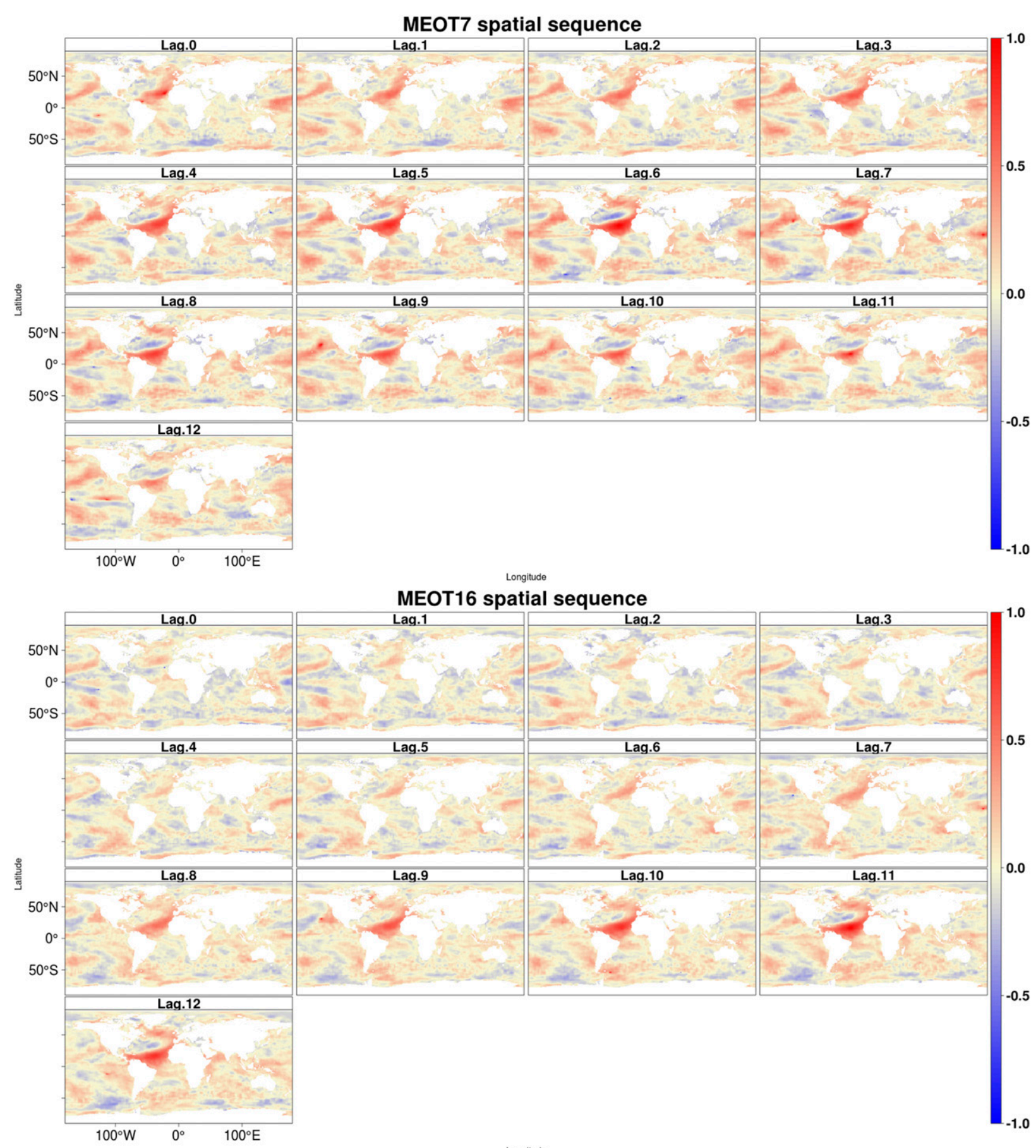

FIG. 4. Temporal map sequences for (top) MEOT7 and (bottom) MEOT16 illustrate the development of the AMM. The peak of the event occurs in MEOT7 around lag 6.

temporal profile correlates the most with Niño-3.4 (0.69) but also displays strong correlation with three other climate indices: PDO (0.56), AMO (0.59), and TSA ( -0.57$)$ (Table 5). The MSSA1 spatial pattern exhibits a spatial configuration that resembles the most an ENSO event, with the entire map sequence exhibiting the pattern of warmENSO-phase El Niño (Fig. S10). The MSSA1 spatialsequence map shows the strengthening of the El Niño event from lag 0 through lag 12 . Thus, evidence indicates that MSSA1 is most strongly related to ENSO but also 
Temporal profiles for MEOT7 and MEOT16

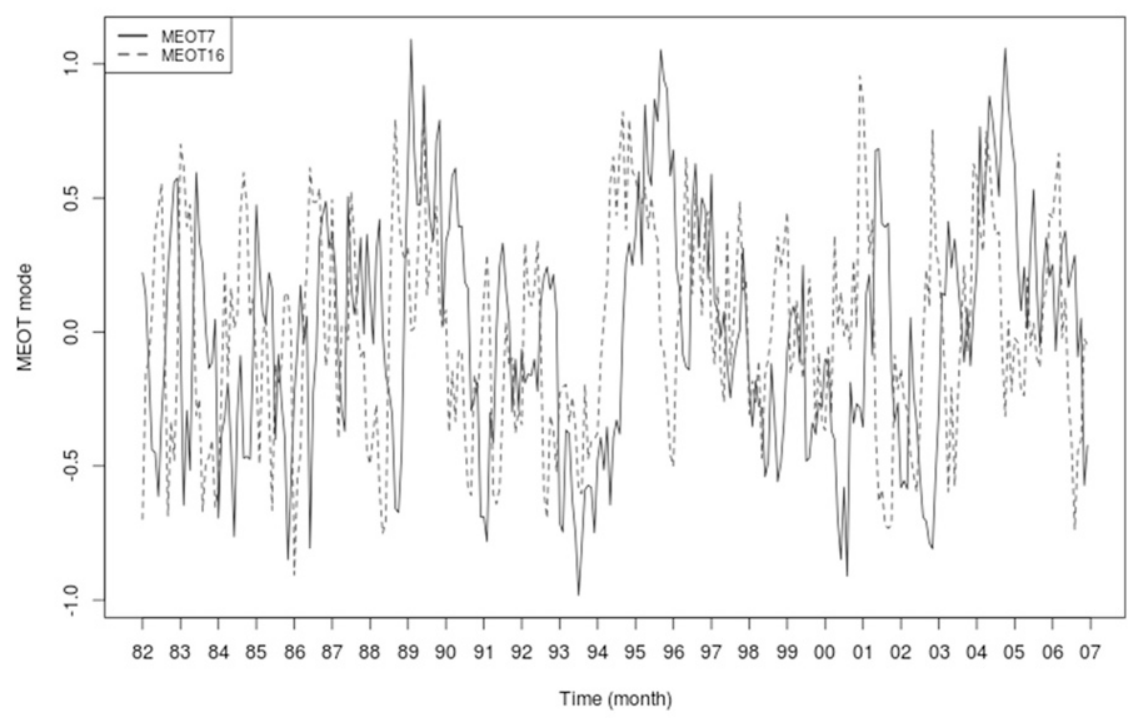

MEOT7 and MEOT16 lag analysis

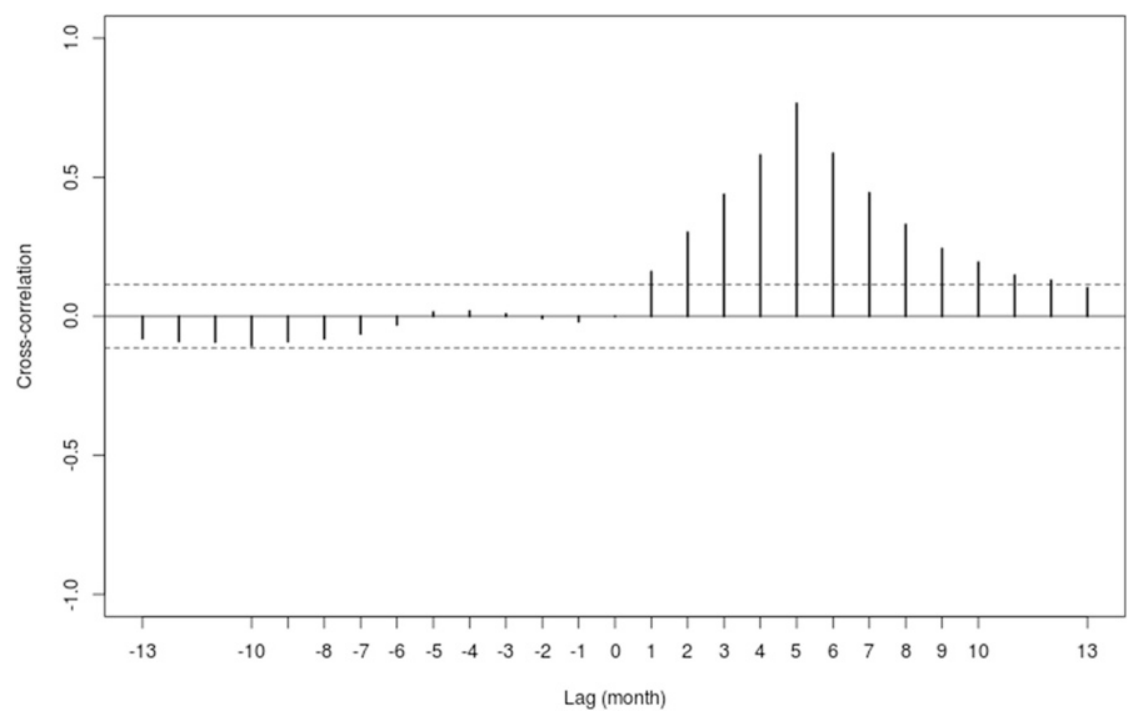

FIG. 5. (bottom) The cross-correlation function between MEOT7 and MEOT16 exhibits a peak of 0.77 at lag 5 and illustrates that MEOT 16 leads MEOT7 by 5 months. (top) The temporal profiles confirm that MEOT7 and MEOT16 have similar temporal patterns. The dashed lines in the bottom graph represent the $95 \%$ confidence interval.

exhibits strong correlations with two other teleconnections (PDO and TSA), suggesting some mixing of modes.

MSSA3 displays the transition from the El Niño conditions characterized by warm SST anomalies along the coast of South America to a normal state with cold waters along South America (Fig. S10). MSSA3 appears to follow MSSA1, and the two components overlap between the first and last three window sequences (lags 11, 12, and 13 from MSSA1 overlap with lags 1, 2, and 3 of MSSA3). MSSA3 correlates the most with the ENSO-related index Niño-3.4 (0.57) and shows less mixing than MSSA1 because all other cross correlations are less than 0.5 , with TNA being the second-strongest correlation $(-0.39)$ (Fig. S12). The lag cross-correlation analysis suggests that MSSA1 and MSSA3 are in quadrature, with the cross-correlation function displaying its strongest absolute value at lag -11 with a correlation of 0.53 (Table 4 and Fig. S11). This indicates that MSSA3 follows MSSA1 by 11 months as visible from the plot of the loadings where MSSA 3 is shifted forward by 11 months in comparison 

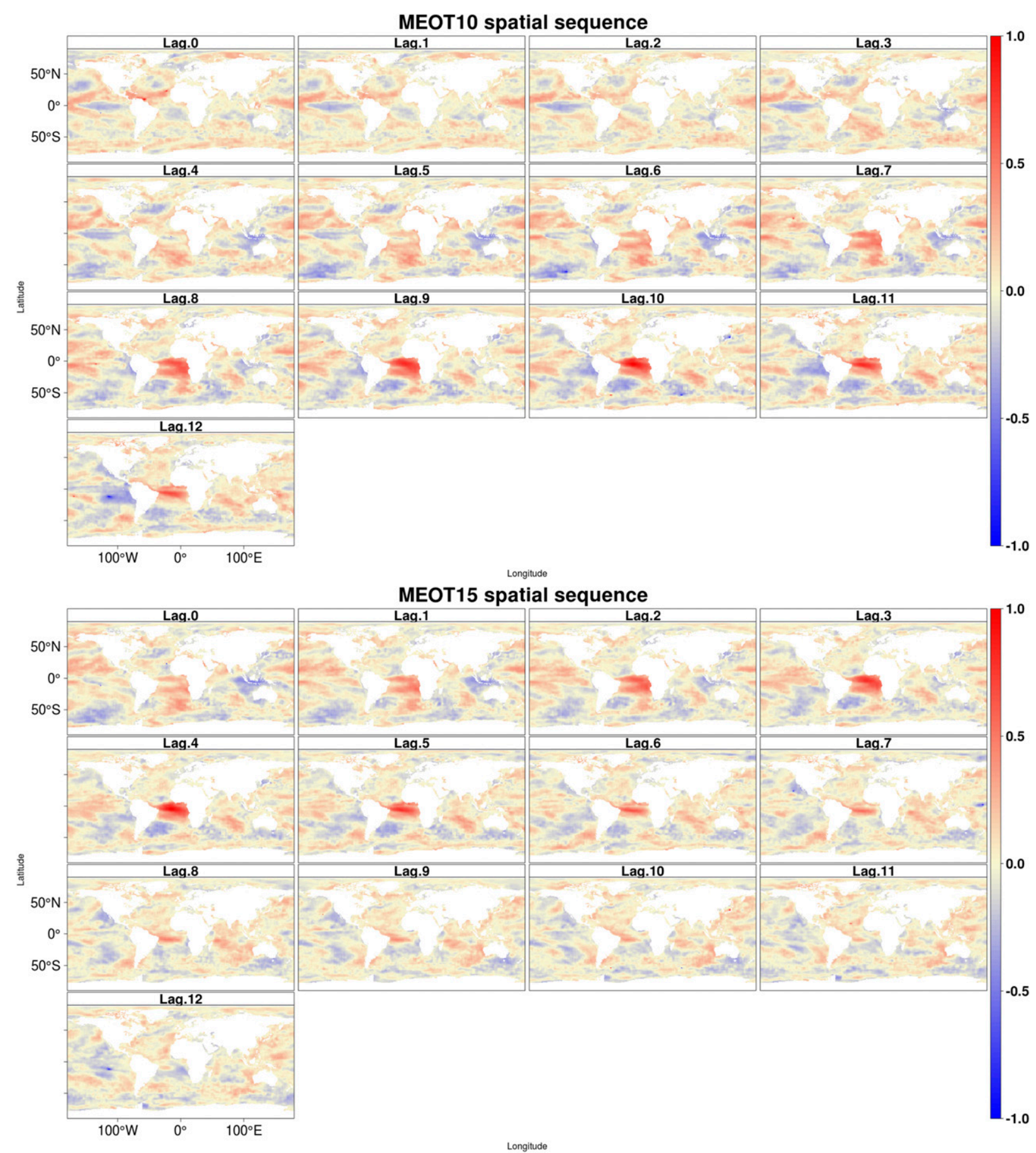

FIG. 6. The temporal map sequences for the MEOT10-MEOT15 pair illustrate the development of the SAOD teleconnection characterized by a gradual warming over the Gulf of Guinea and a cooling at the center of the tropical South Atlantic Gyre.

with MSSA1 (Fig. S11). While the peak of cross correlation falls below the 0.6 threshold and is less pronounced than that found in MEOT quadratures, combined evidence from the lag analyses and the map sequence indicate that the pair relates to the ENSO event.

\section{Discussion}

In the following section, we emphasize and discuss three important points with regard to the results of the MEOT analysis and its properties in general: 1) the 
Temporal profiles for MEOT10 and MEOT15

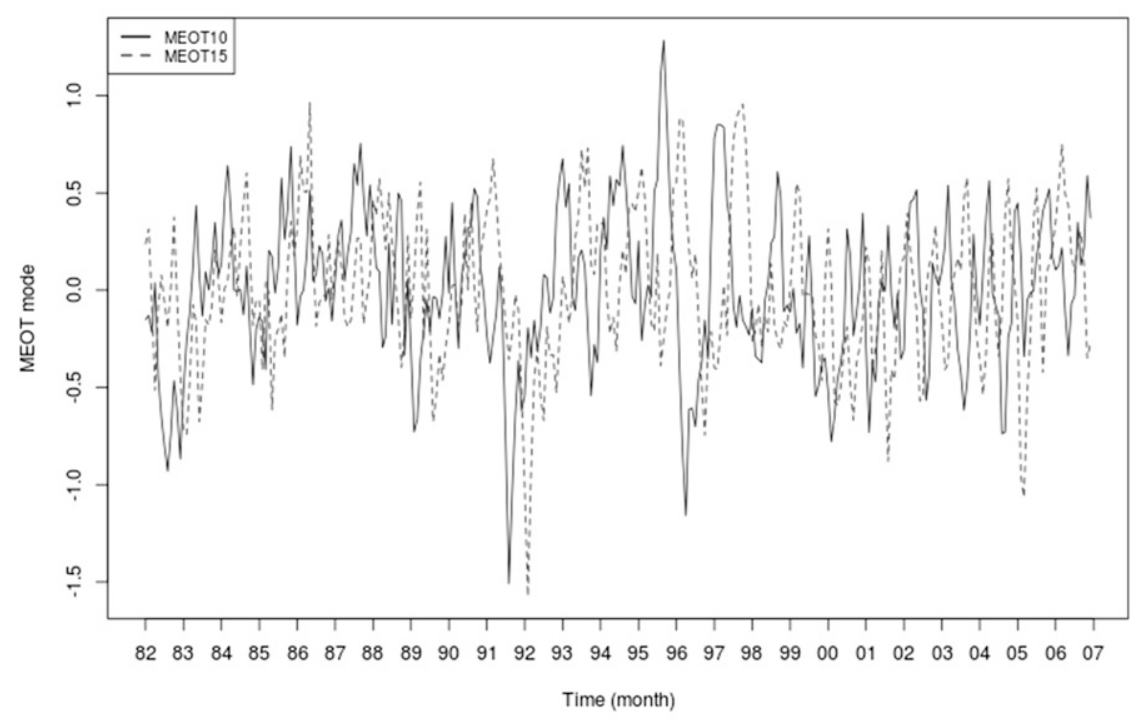

MEOT10 and MEOT15 lag analysis

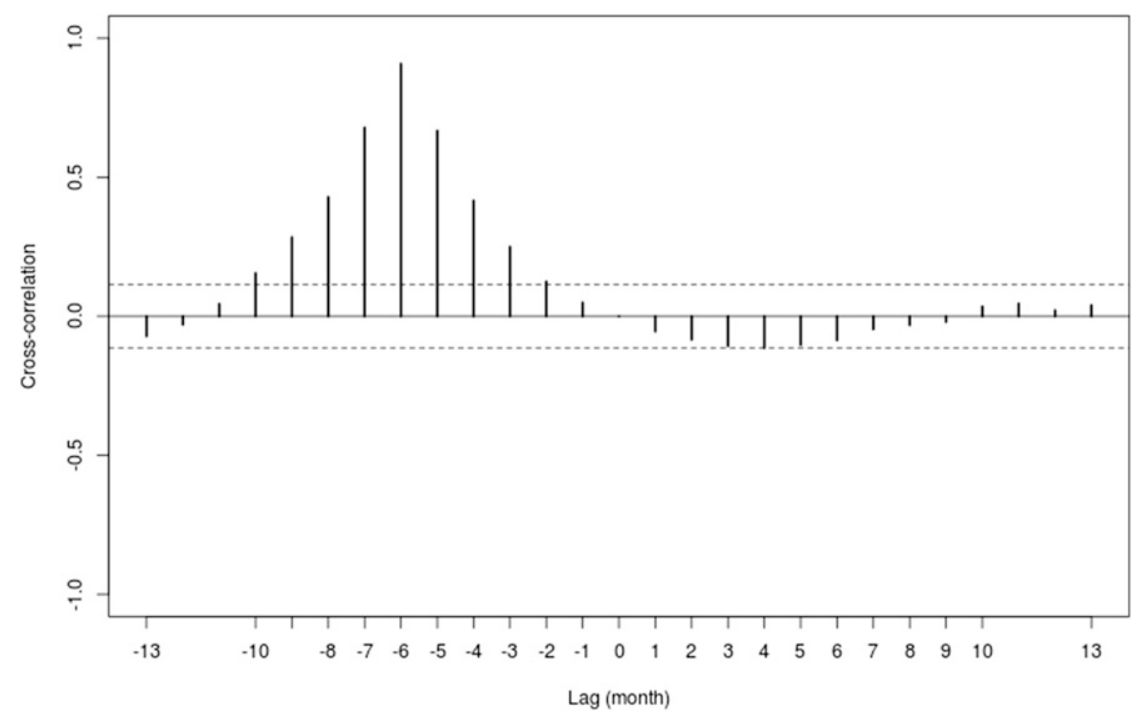

FIG. 7. (top) The temporal profiles of the MEOT10-MEOT15 pair illustrate the similarity in temporal patterns and show that MEOT10 (solid) precedes MEOT15 (dashed) by 6 months. (bottom) The cross-correlation function between MEOT10 and MEOT15 displays a peak of 0.91 at lag -6 .

meaning of quadratures, 2) the effect of lag-window length (embedding dimension), and 3 ) the issue of how it behaves in the presence of red noise.

\section{a. Quadrature in the literature}

In SSA, modes in quadrature correspond to pairs of temporal basis functions with similar temporal patterns shifted in time. The identification of moving modes may be done by searching for quadratures corresponding to cross-correlation functions that exhibit clear peaks. In MSSA, outputs provide both a temporal pattern and a sequence of maps. Two components are in quadrature if they show similar patterns in the temporal dimension and in the space dimension (Jolliffe 2002; Wang 2001). In consequence, for a mode to be in quadrature, a pair of map sequences with similar spatial patterns shifted in time is also required. Thus, in MEOT analysis, components are identified as quadratures if map patterns 


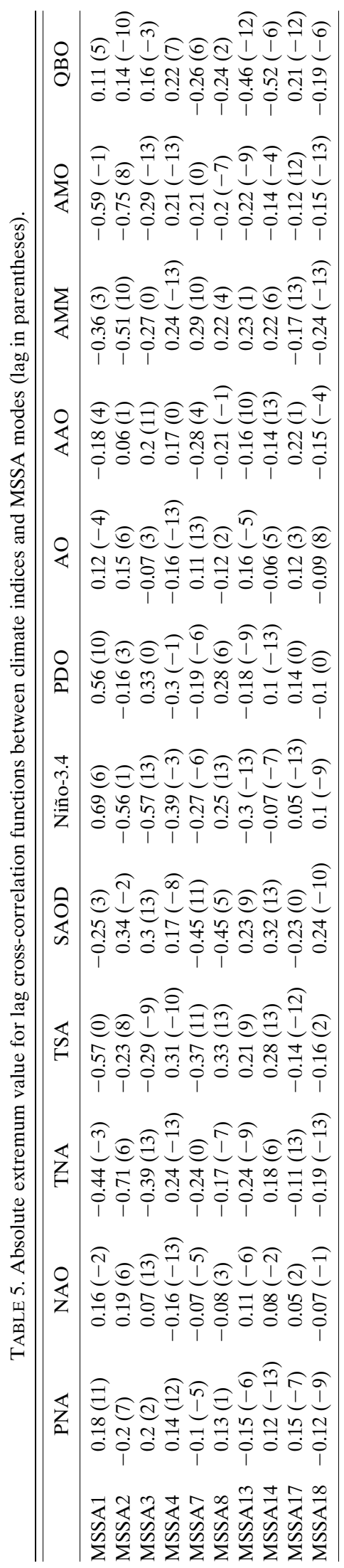

describe the evolution of a climate phenomenon in a series of different phases but also shifted in space in the map sequence. On the basis of this criterion, results provided three clear patterns in quadrature that relate to three known climate teleconnection indices (ENSO, AMM, and SAOD).

\section{b. The effect of lag windows on the MEOT}

The literature reports that MSSA is influenced by the length of the lag window (Ghil et al. 2002). Since the lagwindow length $L$ may affect the results of the MEOT as well, we tested on smaller artificial datasets the effect of the change in the lag window $L$ (embedding dimension) on the detection of phase changes for a given periodic phenomenon. The artificial-image time series dataset was composed of individual images containing 81 pixels in a $9 \times 9$ pattern. Each pixel contained a small independent random component (with a mean of 0 and a standard deviation of 0.0004 ) and one of nine systematic sinusoidal patterns of variation over time. The nine systematic patterns contained an identical sinusoidal pattern with different phase shifts over nine steps (Fig. 8). These were applied equally to groups of $3 \times 3$ pixels such that the wave peaks exhibited a spatiotemporal " $S$ " pattern in the $3 \times 3$ blocks progressing from lower left to upper right. Thus, the full spatiotemporal pattern had a period $P$ of nine time steps. This sequence was repeated 10 times to yield a complete time series of 90 images.

Using this artificial dataset, we conducted MEOT analyses using lag-window lengths of 5, 6, 7, 8, 9, and 18 and found the following:

1) For a lag window of length $>6$ but $\leq 9$, the temporal sinusoidal signal is captured in the first two components that are in quadrature. The spatial pattern shows the development of the phenomenon in two components when the lag window is less than the period of the phenomenon (Figs. 9 and 10).

2) For a lag window of length $\leq 6$, the temporal sinusoidal pattern is captured in the first two components but the original spatial pattern is split in the first two components and cannot be reconstructed from the MEOT spatial patterns (Figs. 9 and 10).

3) For lag-window length $>9$, the spatial pattern is repeated. For instance, with lag-window length of 18, the pattern is shown twice (Figs. 9 and 11).

4) In all cases, the temporal sinusoidal pattern is recovered in the first two temporal MEOT components (Fig. 9).

The artificial dataset suggests that the full spatial map sequence can be recovered whenever the lag window of length $L$ is greater than or equal to 0.75 of the period, or $L=P-(P / 4)$. For the artificial dataset, the period is 9 
a.
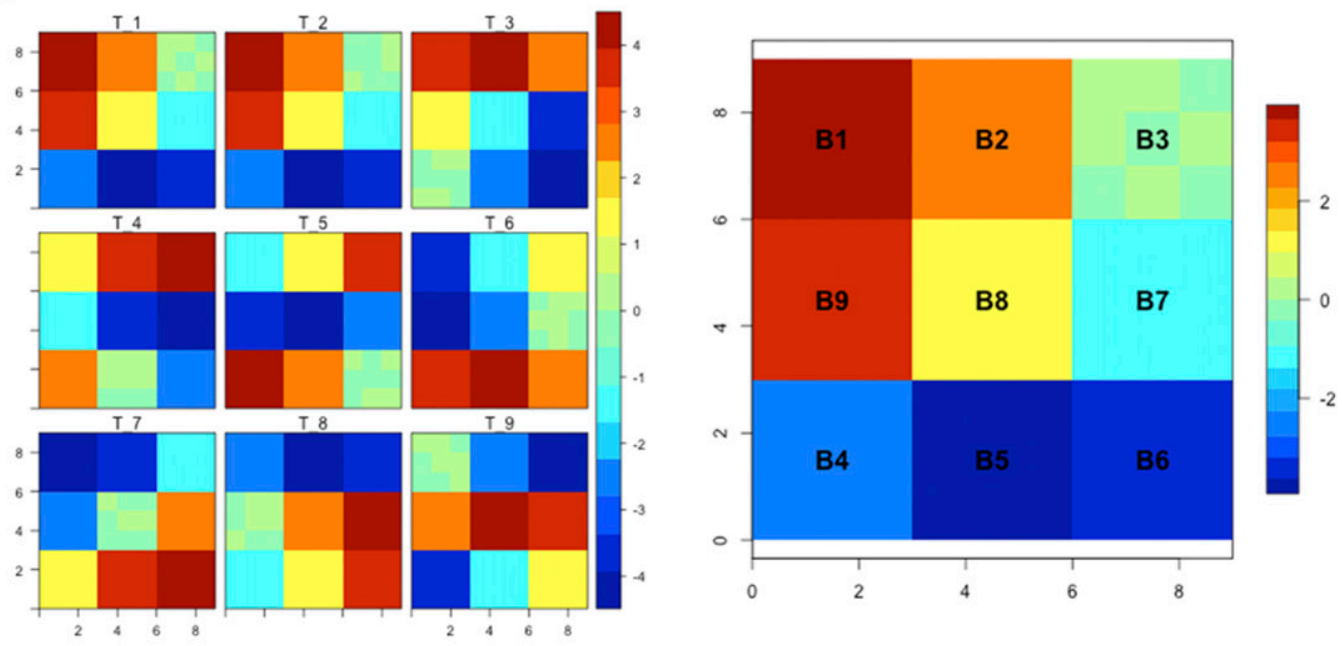

b.

Sinusoidal patterns for the 9 blocks
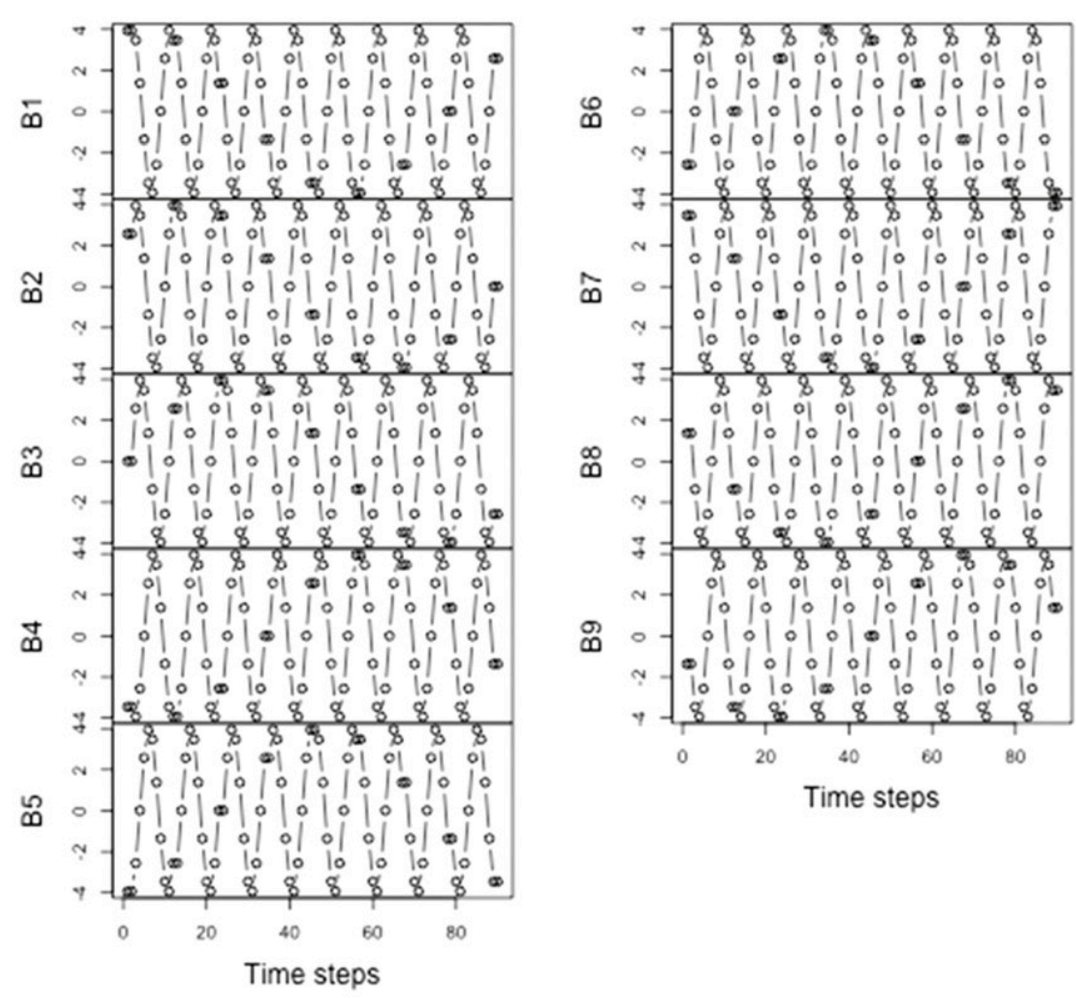

FIG. 8. Synthetic dataset composed of (a) 81 pixels and nine subpatterns, with (b) the associated times series of sinusoidal signal out of phase.

and $L$ must be greater than or equal to 6.75 , that is, at least 7. Furthermore, the lag window has an effect on the length of the temporal MEOT component/index extracted. For instance, if the lag window is 8 , the length of the total time series analyzed will be $90-8+1=83$. Thus, we found that, for a phenomenon to be fully detected spatially (i.e., every spatial map sequence shown), we must use a lag window of at least three-quarters of the period of the cycle.

For the global analysis of SST, we chose a window of 13 months. Thus, in the context of our analysis, this choice implies that we should have been able to uncover events with periods of 17.3 months or greater. The results indicated that the three phenomena uncovered 

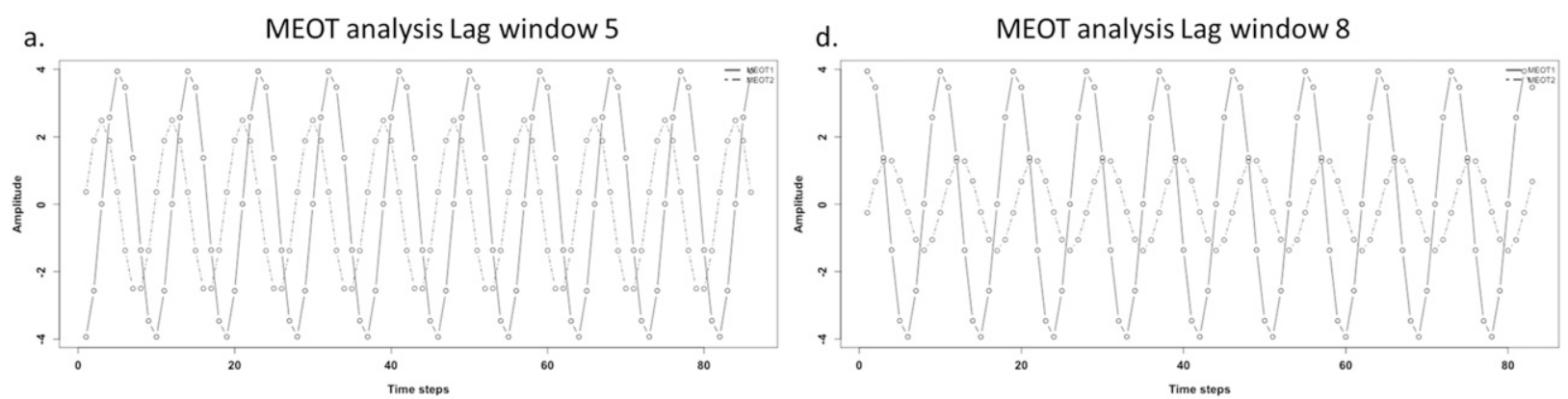

b. $\quad$ MEOT analysis Lag window 6

e.
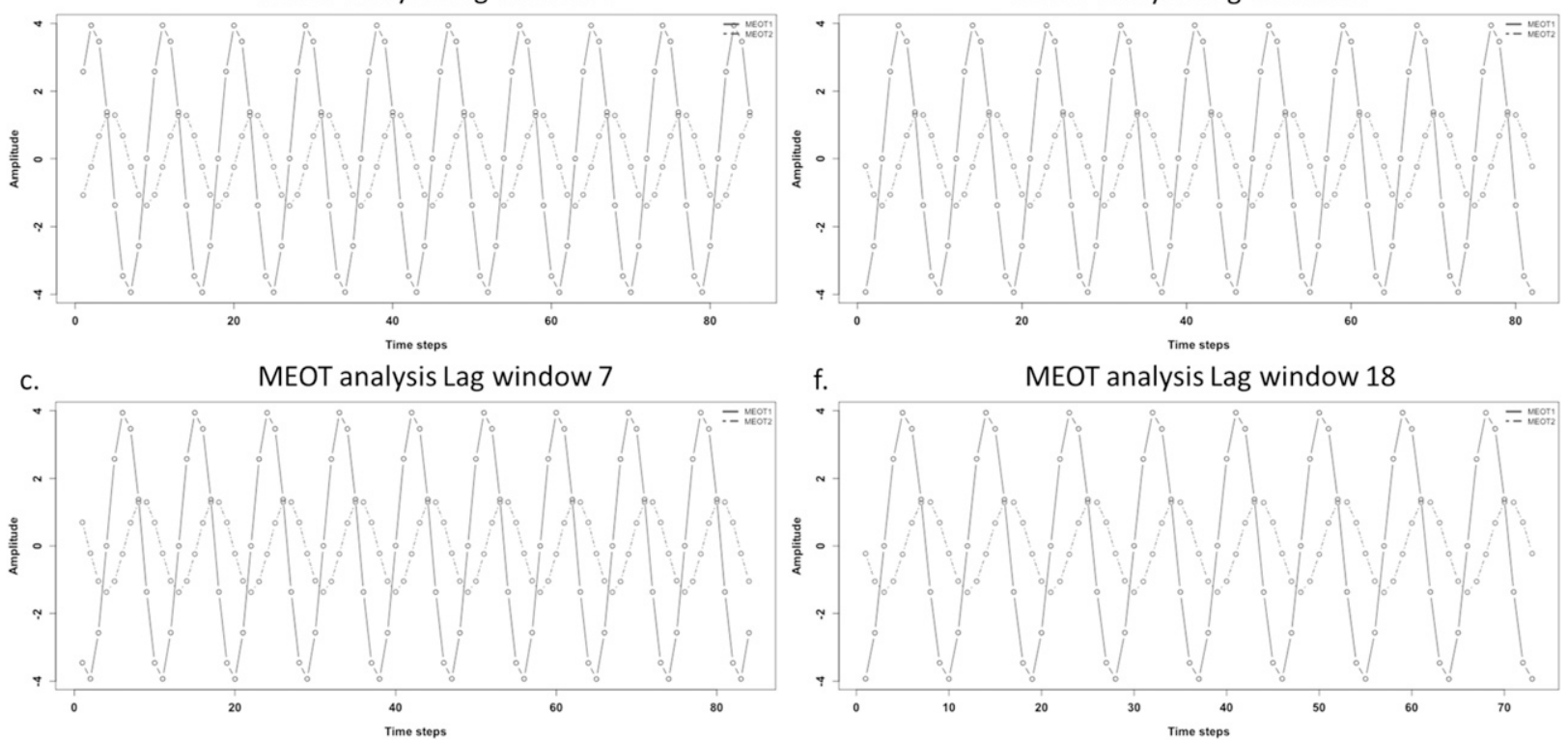

FIG. 9. Temporal patterns for MEOT1 (solid) and MEOT2 (dashed) for lag windows of length 5-9 and 18: In all cases, the temporal pattern is recovered by the first two MEOT temporal profiles.

(ENSO, AMM, and SAOD) show event periods (as opposed to repeat periods) of 24,20 , and 24 months (i.e., quadrature lags of 6,5 , and 6 months) respectively.

\section{c. The behavior of MEOT in the presence of red noise}

The climate literature also raises the issue of red noise when decomposing datasets. To identify teleconnections, we used 12 published indices from NOAA and the literature (Table 1). Most of these indices exhibit high autoregressive lag-1 (AR1) correlations, and entire papers are devoted to the problem of distinguishing components in the presence of red noise (Allen and Robertson 1996; Feldstein 2000; Meinke et al. 2005; Rodionov 2006). To explore empirically how the MEOT algorithm performs in the presence of red noise, we created two small artificial datasets that contain temporal red noise with and without spatial structures. Red noise was added by using a first-lag autoregressive model with AR1 of 0.92 , which corresponds to the AR1 coefficient of the ENSO Niño-3.4 index. For the white-noise component of the AR1 model, we used a mean of 0 and a standard deviation of 1. Using this red-noise base dataset, we generated an additional dataset by adding a spatial trend at every time step. All datasets contain 9 pixels $(3 \times 3$ raster images $)$ with times series of length 45 (Figs. 12a,b).

We found that high temporal cross correlation can occur for MEOT components extracted, but the spatial patterns are not similar (Fig. 12). The spatial patterns of MEOT components did not portray a sequence of moving patterns. We also found that patterns were noisy and did not display coherent spatial structure. Furthermore, in the case of the red-noise dataset with spatial trend, we were unable to recover the original spatial structure (i.e., the spatial trend added) in the MEOT map sequences. We found that occasional high cross correlation may occur among temporal base patterns by chance. In instances in which that occurred, however, they failed to exhibit phase-shifted similarities in their spatial patterns (Figs. 12c,d). In contrast, in the context of the global SST 
a.

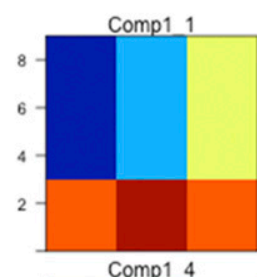

Lag window 5 MEOT1
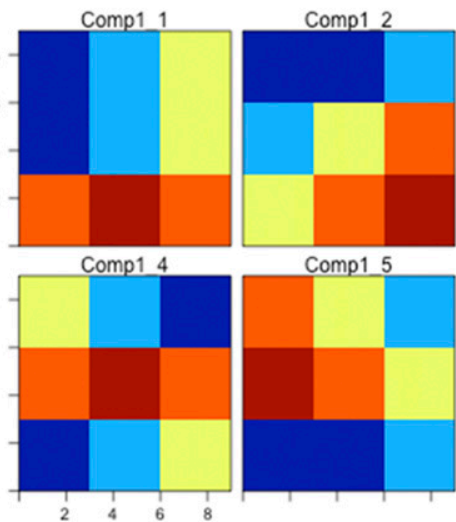

b.
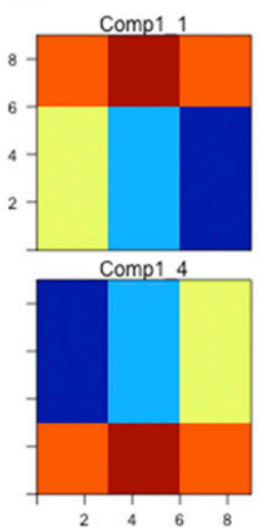

Lag window 6 MEOT1

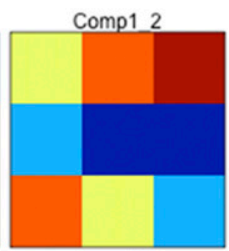

Comp1 5

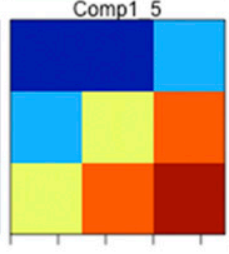

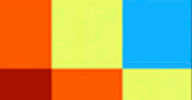
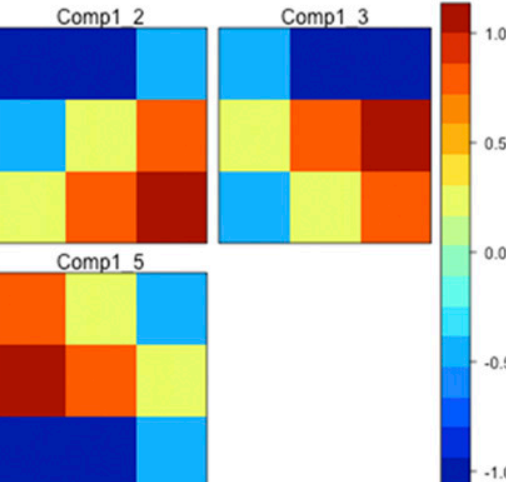

Lag window 7 MEOT1

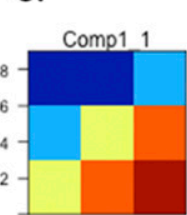

Comp1 5

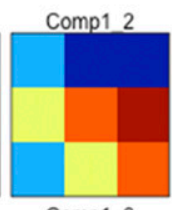

Comp1 6
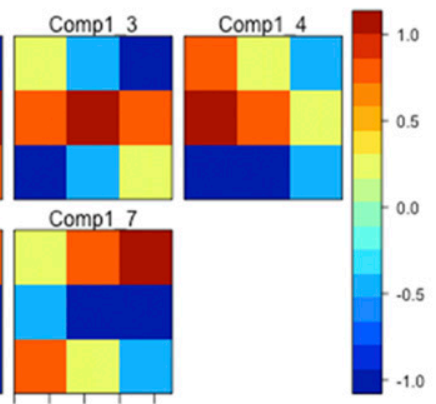

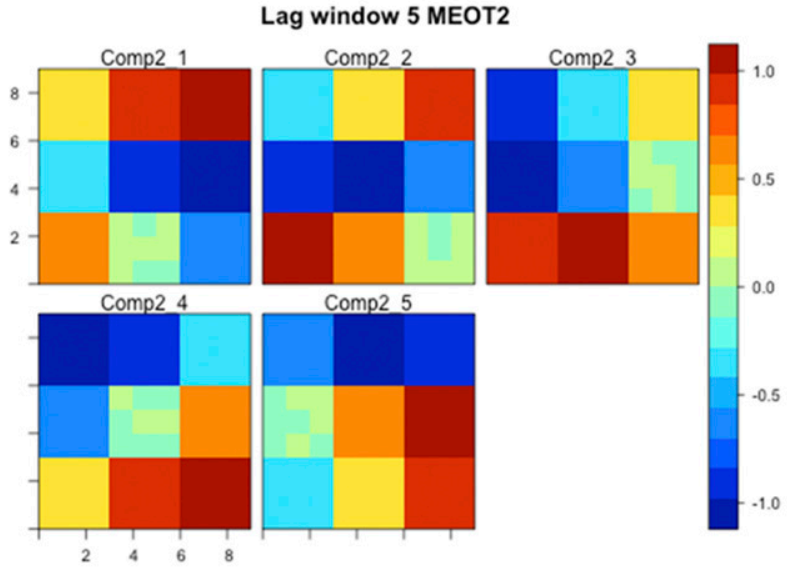

Lag window 6 MEOT2
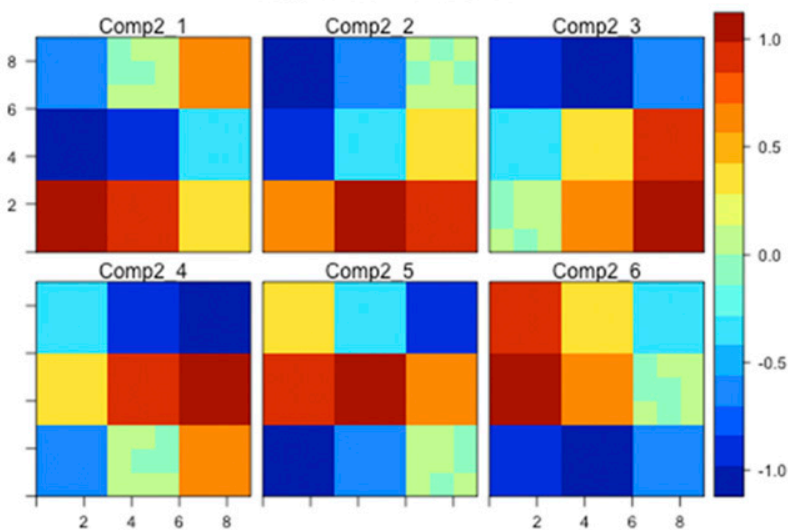

Lag window 7 MEOT2
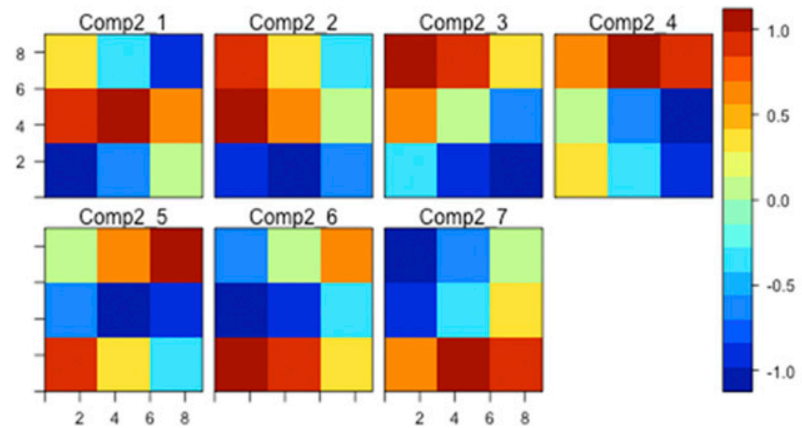

FIG. 10. Spatial patterns for (left) MEOT1 and (right) MEOT2 for lag windows of length (a) 5, (b) 6, and (c) 7. In (a) and (b) the spatial pattern is split between two MEOTs when using a lag window of 5 or 6 but cannot be reconstructed with two components; in (c) with a lag window of 7, the spatial pattern is split between the two components and can be reconstructed.

analysis, we found clear similarity in the spatial patterns in the quadrature pairs as well as development of the phenomena over time. In summary, components uncovered with MEOT from series that possess patterns solely as a result of red noise fail to produce pairs on components that are in quadrature in both space and time.

Note also that, like EOT, MEOT is a brute-force regression technique that is very computationally intensive. Before it can be effectively used as a mainstream technique in climatological research, effective software implementations will need to be developed that can make best use of parallel-processing techniques and highperformance computer clusters or cloud computing.

\section{Conclusions}

This research introduced multichannel empirical orthogonal teleconnection analysis as an extension of the 
a.

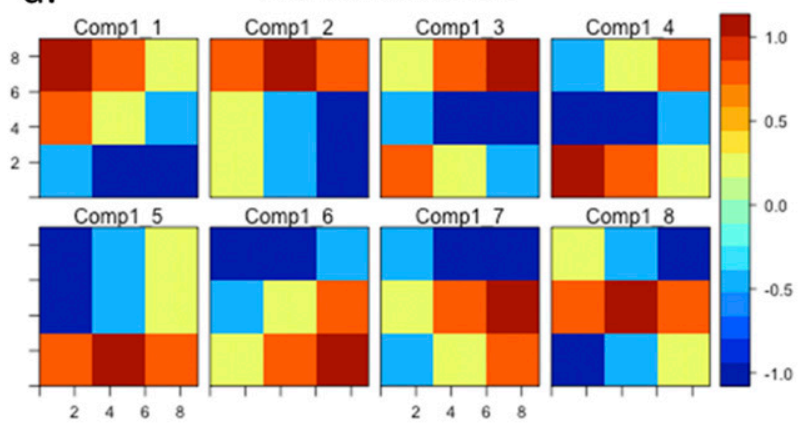

b.

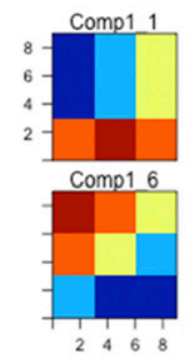

c.

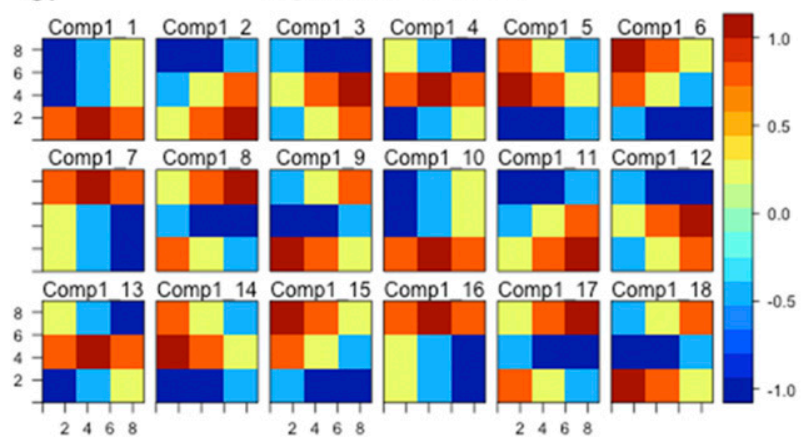

Lag window 9 MEOT1
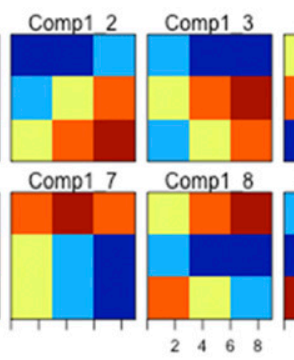

\begin{tabular}{lllll}
2 & 4 & 6 & 1 \\
\hline & 4 & 8
\end{tabular}
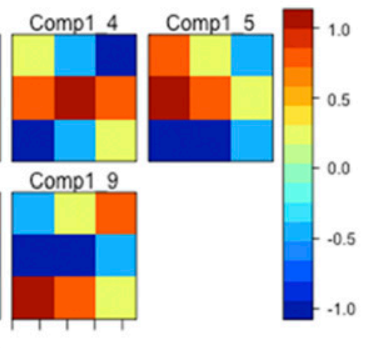

Lag window 18 MEOT1
FIG. 11. Spatial patterns for (left) MEOT1 and (right) MEOT2 for a lag windows of length (a) 8, (b) 9, and (c) 18. In (a) with lag window 8 , eight phases of nine are represented in each component, and both can be used to reconstruct the full pattern; In (b), all of the phases are represented in MEOTs with lag window 9; In (c) with lag window 18, the nine phases of the spatial patterns are fully represented in each component but the original cycle is repeated.

empirical orthogonal teleconnection analysis to extract climate modes of variability coevolving in time and space. Extraction and identification of such climate modes are critical to improve our understanding of the Earth system and provide predictions to inform on the potential direct or indirect impacts of these phenomena on society (e.g., drought, fire, and flooding). Using a global SST dataset, we explore similarities and differences between MEOT and MSSA in the extraction of quadratures and their interpretation. Results show that the MEOT method was able to extract several climate modes coevolving in space and time. We identified three modes in quadratures, in both space and time, related to the ENSO, AMM, and SAOD teleconnections with event periods (as opposed to repeat cycles) of 24, 20, and
24 months, respectively. Findings indicate that both MSSA and MEOT analyses were able to extract several climate modes coevolving in space and time, but results differed in both the number and the clarity of the patterns. For the MEOTs we obtained three modes in quadratures related to the ENSO, SAOD, and AMM teleconnections, whereas for the MSSA four quadratures were extracted from which we related two to the ENSO and QBO teleconnections. MSSA modes and quadratures display mixing of climate modes of variability, with the two remaining quadratures (MSSA7MSSA8 and MSSA16-MSSA17) being difficult to identify. For instance, while there is strong evidence that the MSSA1-MSSA3 quadrature relates to the ENSO event, MSSA1 also exhibits strong correlation with 
a.

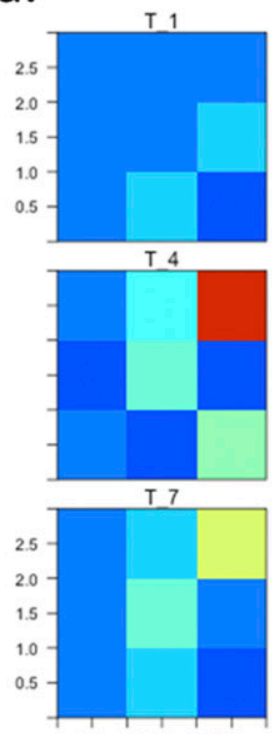

$0.5 \quad 1.0 \quad 1.5 \quad 2.02 .5$
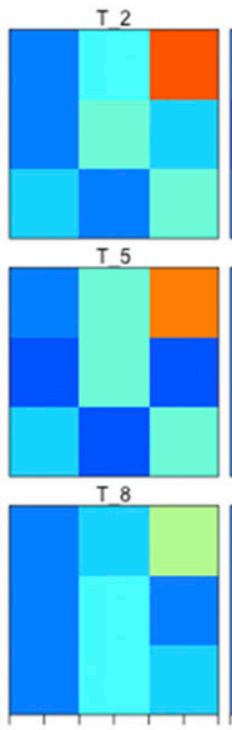
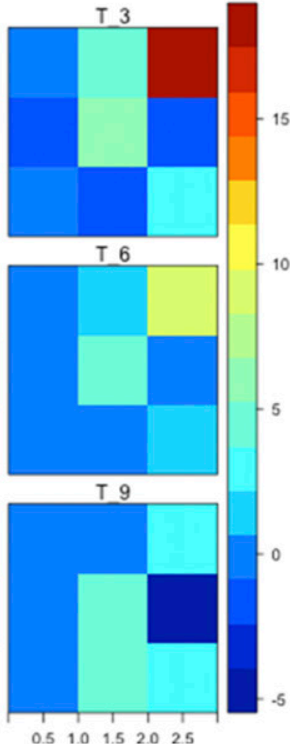

b.

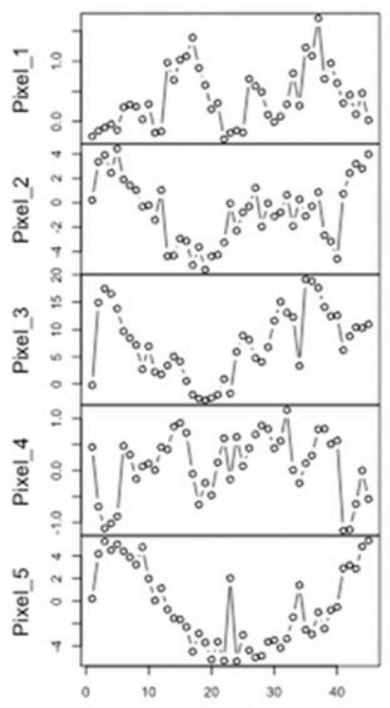

Red noise patterns for the 9 pixels

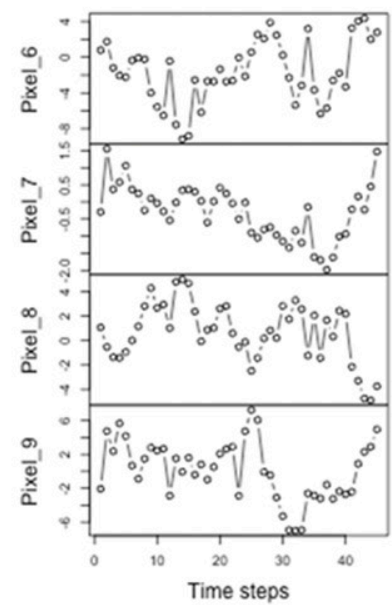

C.

Temporal components for Lag window 9 MEOT analysis

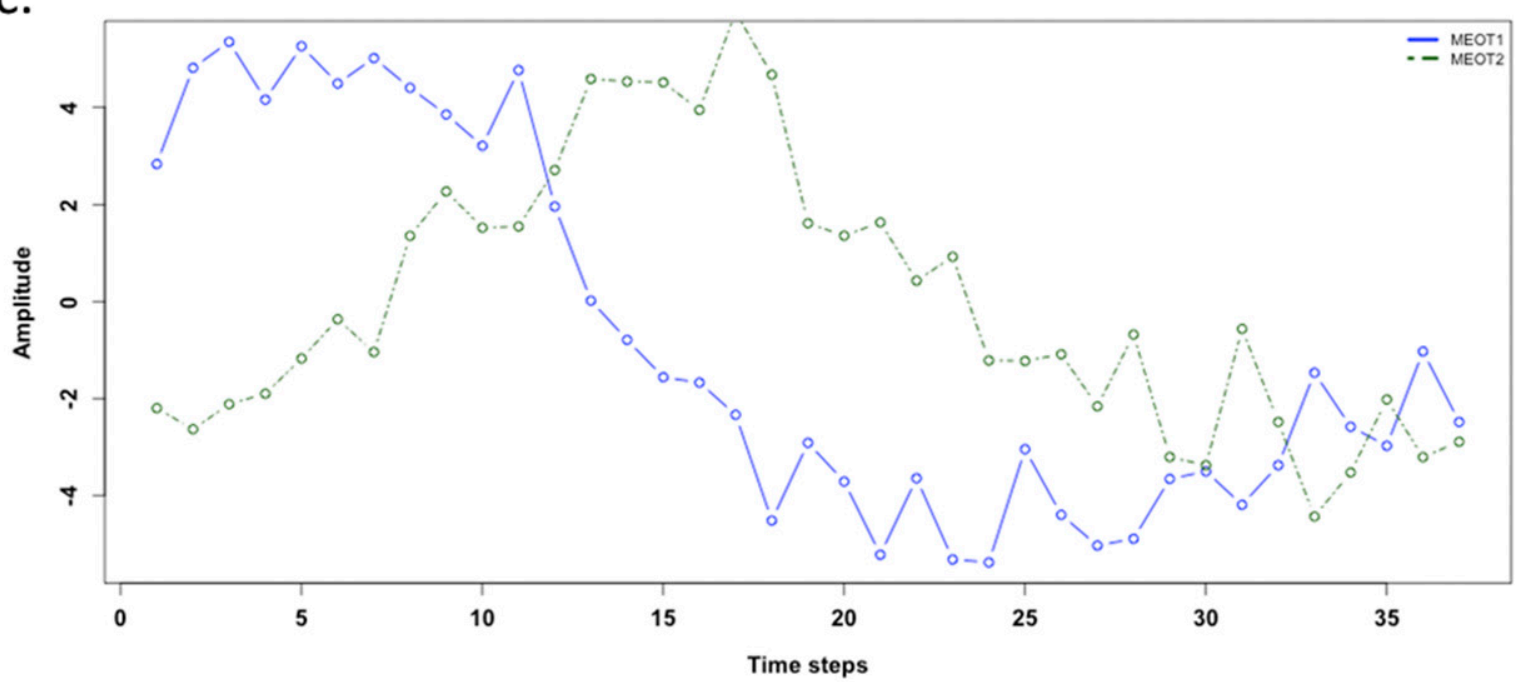

d.
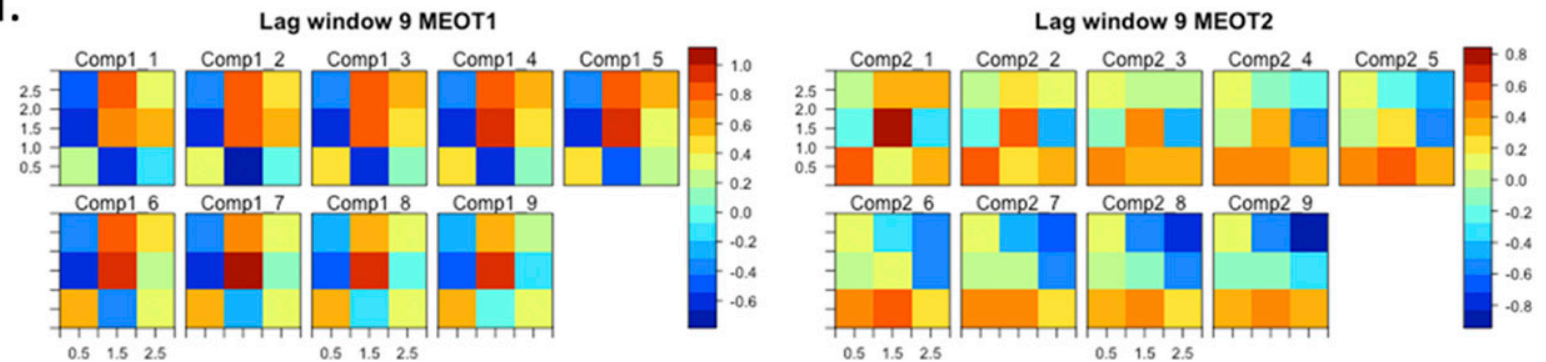

FIG. 12. (a) Synthetic datasets for red noise with the first nine spatial patterns and spatial trend. (b) The dataset comprises time series for nine pixels and shows that the spatial coherence is quickly lost in the presence of red noise. (c), (d) It is seen that the original signal is not recovered when MEOT analysis is carried out with a lag window of length 9. 
PDO, AMO, and TSA modes. Such mixed patterns render the identification of individual teleconnection arduous and decrease their usefulness in climate prediction (Kim and Wu 1999; van den Dool 2007). In contrast, MEOT modes display less mixing of modes, and, when they do, their spatial patterns clearly eliminate any confusion. For instance, the MEOT10MEOT5 and MEOT7-MEOT16 pairs display strong correlations with the TNA and TSA indices, respectively, but the spatial patterns in the sequence of maps unambiguously relate them to the AMM and the SAOD modes, respectively.

Findings indicate that MEOT analysis is able to extract pairs of modes that correspond to phenomena coevolving in space and time. Results also indicate that the new MEOT method differs from MSSA in extracting oscillatory patterns in both the number of quadratures and relation to known indices. MSSA extracted more quadratures but displayed more mixing. Given MEOT's special quality that it relaxes the biorthogonality constraint that is associated with techniques such as MSSA, these results suggest that further research is warranted to assess its competitive strengths relative to alternative solutions such as rotated MSSA.

Acknowledgments. This paper developed out of ongoing research on time series analysis at the Clark Labs of Clark University. Clark Labs also facilitated this work by providing implementation of MEOT methods in the GIS software Idrisi (now TerrSet) and its vertical application known as the Earth Trend Modeler. Statistical analyses were also conducted using SPSS and the R software packages and computing platform. The authors thank Ronald J. Eastman for his help in improving the manuscript.

\section{REFERENCES}

Allen, M. R., and A. W. Robertson, 1996: Distinguishing modulated oscillations from coloured noise in multivariate datasets. Climate Dyn., 12, 775-784, doi:10.1007/s003820050142.

Berry, B. J., 1964: Approaches to regional analysis: A synthesis. Ann. Assoc. Amer. Geogr., 54, 2-11, doi:10.1111/ j.1467-8306.1964.tb00469.x.

Broomhead, D. S., and G. P. King, 1986: Extracting qualitative dynamics from experimental data. Physica D, 20, 217-236, doi:10.1016/0167-2789(86)90031-X.

— London, 423A, 103-121, doi:10.1098/rspa.1989.0044.

Carissimo, B. C., A. H. Oort, and T. H. Vonder Haar, 1985: Estimating the meridional energy transports in the atmosphere and ocean. J. Phys. Oceanogr., 15, 82-91, doi:10.1175/ 1520-0485(1985)015<0082:ETMETI>2.0.CO;2.

Chase, T. N., R. A. Pielke, and R. Avissar, 2006: Teleconnections in the Earth system. Encyclopedia of Hydrological Sciences. M. G. Anderson et al., Eds., John Wiley and Sons, 15:183, doi:10.1002/0470848944.hsa190.
Chiang, J. C. H., and D. J. Vimont, 2004: Analogous Pacific and Atlantic meridional modes of tropical atmosphere-ocean variability. J. Climate, 17, 4143-4158, doi:10.1175/JCLI4953.1.

Chiew, F. H., and T. A. McMahon, 2002: Global ENSO-streamflow teleconnection, streamflow forecasting and interannual variability. Hydrol. Sci. J., 47, 505-522, doi:10.1080/ 02626660209492950.

Compagnucci, R. H., D. Araneo, and P. O. Canziani, 2001: Principal sequence pattern analysis: A new approach to classifying the evolution of atmospheric systems. Int. J. Climatol., 21, 197217, doi:10.1002/joc.601.

Cook, E. R., R. D. D'Arrigo, and M. E. Mann, 2002: A well-verified, multiproxy reconstruction of the winter North Atlantic Oscillation index since A.D. 1400. J. Climate, 15, 1754-1764, doi:10.1175/1520-0442(2002)015<1754:AWVMRO>2.0.CO;2.

Dai, A., K. E. Trenberth, and T. Qian, 2004: A global dataset of Palmer drought severity index for 1870-2002: Relationship with soil moisture and effects of surface warming. J. Hydrometeor., 5, 1117-1130, doi:10.1175/JHM-386.1.

Deser, C., M. A. Alexander, S.-P. Xie, and A. S. Phillips, 2010: Sea surface temperature variability: Patterns and mechanisms. Аnnu. Rev. Mar. Sci., 2, 115-143, doi:10.1146/ annurev-marine-120408-151453.

Dirmeyer, P., and J. Shukla, 1993: Observational and modeling studies of the influence of soil moisture anomalies on atmospheric circulation (review). Prediction of Interannual Climate Variations, J. Shukla, Ed., NATO ASI Series, Vol. 6, Springer, 1-23, doi:10.1007/978-3-642-76960-3_1.

Elsner, J. B., and A. A. Tsonis, 2013: Singular Spectrum Analysis: A New Tool in Time Series Analysis. Springer Science and Business Media, 164 pp.

Enfield, D. B., A. M. Mestas-Nuñez, D. A. Mayer, and L. CidCerrano, 1999: How ubiquitous is the dipole relationship in tropical Atlantic sea surface temperatures? J. Geophys. Res., 104, 7841-7848, doi:10.1029/1998JC900109.

,$- \ldots$, and P. J. Trimble, 2001: The Atlantic multidecadal oscillation and its relation to rainfall and river flows in the continental U.S. Geophys. Res. Lett., 28, 2077-2080, doi:10.1029/2000GL012745.

Feldstein, S. B., 2000: The timescale, power spectra, and climate noise properties of teleconnection patterns. J. Climate, 13, 4430-4440, doi:10.1175/1520-0442(2000)013<4430: TTPSAC $>2.0 . C O ; 2$.

Florenchie, P., J. R. Lutjeharms, C. J. C. Reason, S. Masson, and M. Rouault, 2003: The source of Benguela Niños in the South Atlantic Ocean. Geophys. Res. Lett., 30, 1505, doi:10.1029/ 2003 GL017172.

Ghil, M., and Coauthors, 2002: Advanced spectral methods for climatic time series. Rev. Geophys., 40, 1003, doi:10.1029/ 2000RG000092.

Graham, N. E., J. Michaelsen, and T. P. Barnett, 1987a: An investigation of the El Niño-Southern Oscillation cycle with statistical models: 1. Predictor field characteristics. J. Geophys. Res., 92, 14 251-14 270, doi:10.1029/JC092iC13p14251.

,-- , and $-1987 \mathrm{~b}$ : An investigation of the El NiñoSouthern Oscillation cycle with statistical models: 2. Model results. J. Geophys. Res., 92, 14271-14289, doi:10.1029/ JC092iC13p14271.

Groth, A., and M. Ghil, 2011: Multivariate singular spectrum analysis and the road to phase synchronization. Phys. Rev., 84E, 036206, doi:10.1103/PhysRevE.84.036206.

Huang, B., Z. Zhen, J. Kinter III, Z. Wu, and A. Kumar, 2012: Connection of stratospheric QBO with global atmospheric 
general circulation and tropical SST. Part I: Methodology and composite life cycle. Climate Dyn., 38, 1-23, doi:10.1007/ s00382-011-1250-7.

IPCC, 2007: Climate Change 2007: The Physical Science Basis. Cambridge University Press, $996 \mathrm{pp}$.

Jolliffe, I. T., 2002: Principal component analysis and factor analysis. Principal Component Analysis, 2nd ed. Springer-Verlag, $150-166$.

—- M. Uddin, and S. Vines, 2002: Simplified EOFs-Three alternatives to rotation. Climate Res., 20, 271-279, doi:10.3354/ cr020271.

Jones, P. D., T. Jonsson, and D. Wheeler, 1997: Extension to the North Atlantic Oscillation using early instrumental pressure observations from Gibraltar and south-west Iceland. Int. J. Climatol., 17, 1433-1450, doi:10.1002/(SICI)1097-0088(19971115)17:13<1433:: AID-JOC203>3.0.CO;2-P.

Keppenne, C. L., and M. Ghil, 1992: Adaptive filtering and prediction of the Southern Oscillation index. J. Geophys. Res., 97, 20 449-20 454, doi:10.1029/92JD02219.

Kim, K.-Y., and Q. Wu, 1999: A comparison study of EOF techniques: Analysis of nonstationary data with periodic statistics. J. Climate, 12, 185-199, doi:10.1175/1520-0442-12.1.185.

Knight, J. R., R. J. Allan, C. K. Folland, M. Vellinga, and M. E. Mann, 2005: A signature of persistent natural thermohaline circulation cycles in observed climate. Geophys. Res. Lett., 32, L20708, doi:10.1029/2005GL024233.

Kossin, J. P., and D. J. Vimont, 2007: A more general framework for understanding Atlantic hurricane variability and trends. Bull. Amer. Meteor. Soc., 88, 1767-1781, doi:10.1175/ BAMS-88-11-1767.

Latif, M., and N. E. Graham, 1992: How much predictive skill is contained in the thermal structure of an oceanic GCM? J. Phys. Oceanogr., 22, 951-962, doi:10.1175/1520-0485(1992)022<0951: $\mathrm{HMPSIC}>2.0 . \mathrm{CO} ; 2$.

Lau, K.-M., and P. Chan, 1986: Aspects of the 40-50 day oscillation during the northern summer as inferred from outgoing longwave radiation. Mon. Wea. Rev., 114, 1354-1367, doi:10.1175/ 1520-0493(1986)114<1354:AOTDOD>2.0.CO;2.

Lee, Y.-A., 2002: A T-EOF based prediction method. J. Climate, 15, 226-234, doi:10.1175/1520-0442(2002)015<0226: ATEBPM $>2.0 . \mathrm{CO} ; 2$.

Liu, Z., and M. Alexander, 2007: Atmospheric bridge, oceanic tunnel, and global climatic teleconnections. Rev. Geophys., 45, RG2005, doi:10.1029/2005RG000172.

Mann, M. E., and J. Park, 1999: Oscillatory spatiotemporal signal detection in climate studies: A multiple-taper spectral domain approach. Advances in Geophysics, Vol. 41, Academic Press, 1-131, doi:10.1016/S0065-2687(08)60026-6.

Mantua, N. J., S. R. Hare, Y. Zhang, J. M. Wallace, and R. C. Francis, 1997: A Pacific interdecadal climate oscillation with impacts on salmon production. Bull. Amer. Meteor. Soc., 78, 1069-1079, doi:10.1175/1520-0477(1997)078<1069: APICOW $>2.0 . \mathrm{CO} ; 2$

Meinke, H., P. deVoil, G. L. Hammer, S. Power, R. Allan, R. C. Stone, C. Folland, and A. Potgieter, 2005: Rainfall variability at decadal and longer time scales: Signal or noise? J. Climate, 18, 89-96, doi:10.1175/JCLI-3263.1.

Mestas-Nuñez, A. M., 2000: Orthogonality properties of rotated empirical modes. Int. J. Climatol., 20, 1509-1516, doi:10.1002/ 1097-0088(200010)20:12<1509::AID-JOC553>3.0.CO;2-Q.

Moron, V., R. Vautard, and M. Ghil, 1998: Trends, interdecadal and interannual oscillations in global sea-surface temperatures. Climate Dyn., 14, 545-569, doi:10.1007/s003820050241.
Namias, J., 1981: Teleconnections of 700mb height anomalies for the Northern Hemisphere. Scripps Institution of Oceanography Marine Life Research Program California Cooperative Oceanic Fisheries Investigations Atlas 29, 265 pp.

Nigam, S., and S. Baxter, 2014: Teleconnections. Encyclopedia of Atmospheric Sciences, 2nd ed. G. North, J. Pyle, and F. Zhang, Eds., Academic Press, 90-109.

Nnamchi, H. C., and J. Li, 2011: Influence of the South Atlantic Ocean dipole on West African summer precipitation. J. Climate, 24, 1184-1197, doi:10.1175/2010JCLI3668.1.

,-- , and R. N. C. Anyadike, 2011: Does a dipole mode really exist in the South Atlantic Ocean? J. Geophys. Res., 116, D15104, doi:10.1029/2010JD015579.

Plaut, G., and R. Vautard, 1994: Spells of low-frequency oscillations and weather regimes in the Northern Hemisphere. $J$. Atmos. Sci., 51, 210-236, doi:10.1175/1520-0469(1994)051<0210: SOLFOA $>2.0 . \mathrm{CO} ; 2$.

Potter, C., S. Klooster, M. Steinbach, P. Tan, V. Kumar, S. Shekhar, R. Nemani, and R. Myneni, 2003: Global teleconnections of climate to terrestrial carbon flux. J. Geophys. Res., 108, 4556, doi:10.1029/2002JD002979.

Preisendorfer, R. W., and C. D. Mobley, 1988: Principal Component Analysis in Meteorology and Oceanography. Elsevier, 425 pp.

Reynolds, R. W., N. A. Rayner, T. M. Smith, D. C. Stokes, and W. Wang, 2002: An improved in situ and satellite SST analysis for climate. J. Climate, 15, 1609-1625, doi:10.1175/ 1520-0442(2002)015<1609:AIISAS > 2.0.CO;2.

_, T. M. Smith, C. Liu, D. B. Chelton, K. S. Casey, and M. G. Schlax, 2007: Daily high-resolution-blended analyses for sea surface temperature. J. Climate, 20, 5473-5496, doi:10.1175/ 2007JCLI1824.1.

Richman, M. B., 1986: Rotation of principal components. J. Climatol., 6, 293-335, doi:10.1002/joc.3370060305.

Rodionov, S. N., 2006: Use of prewhitening in climate regime shift detection. Geophys. Res. Lett., 33, L12707, doi:10.1029/ 2006 GL025904.

Rosenzweig, C., and D. Hillel, 2008: Climate Variability and the Global Harvest: Impacts of El Niño and Other Oscillations on Agroecosystems. Oxford University Press, $280 \mathrm{pp}$.

Rotstayn, L. D., M. A. Collier, M. R. Dix, Y. Feng, H. B. Gordon, S. P. O'Farrell, I. N. Smith, and J. Syktus, 2010: Improved simulation of Australian climate and ENSO-related rainfall variability in a global climate model with an interactive aerosol treatment. Int. J. Climatol., 30, 1067-1088, doi:10.1002/ joc. 1952.

Schnur, R., G. Schmitz, N. Grieger, and H. von Storch, 1993: Normal modes of the atmosphere as estimated by principal oscillation patterns and derived from quasigeostrophic theory. J. Atmos. Sci., 50, 2386-2400, doi:10.1175/1520-0469(1993)050<2386: NMOTAA $>2.0 . \mathrm{CO} ; 2$.

Shukla, J., 1984: Predictability of time averages: Part II: The influence of the boundary forcings. Problems and Prospects in Long and Medium Range Weather Forecasting, D. M. Burridge and E. Källen, Eds., Springer, 155-206.

Smith, T. M., and R. W. Reynolds, 2005: A global merged land-airsea surface temperature reconstruction based on historical observations (1880-1997). J. Climate, 18, 2021-2036, doi:10.1175/JCLI3362.1.

Smoliak, B. V., and J. M. Wallace, 2015: On the leading patterns of Northern Hemisphere sea level pressure variability. J. Atmos. Sci., 72, 3469-3486, doi:10.1175/JAS-D-14-0371.1.

Trenberth, K. E., D. P. Stepaniak, and J. M. Caron, 2000: The global monsoon as seen through the divergent atmospheric 
circulation. J. Climate, 13, 3969-3993, doi:10.1175/ 1520-0442(2000)013<3969:TGMAST>2.0.CO;2.

_ J. M. Caron, D. P. Stepaniak, and S. Worley, 2002: Evolution of El Niño-Southern Oscillation and global atmospheric surface temperatures. J. Geophys. Res., 107, doi:10.1029/ 2000JD000298.

van den Dool, H., 2007: Empirical Methods in Short-Term Climate Prediction. Oxford University Press, 240 pp.

— S. Saha, and Å. Johansson, 2000: Empirical orthogonal teleconnections. J. Climate, 13, 1421-1435, doi:10.1175/ 1520-0442(2000)013<1421:EOT>2.0.CO;2.

Vautard, R., P. Yiou, and M. Ghil, 1992: Singular-spectrum analysis: A toolkit for short, noisy chaotic signals. Physica $D, \mathbf{5 8}$, 95-126, doi:10.1016/0167-2789(92)90103-T.

— - G. Plaut, R. Wang, and G. Brunet, 1999: Seasonal prediction of North American surface air temperatures using space-time principal components. J. Climate, 12, 380-394, doi:10.1175/ 1520-0442(1999)012<0380:SPONAS > 2.0.CO;2.

Wallace, J. M., and D. S. Gutzler, 1981: Teleconnections in the geopotential height field during the Northern Hemisphere winter. Mon. Wea. Rev., 109, 784-812, doi:10.1175/ 1520-0493(1981)109<0784:TITGHF>2.0.CO;2.

Wang, R., 2001: Prediction of seasonal climate in a lowdimensional phase space derived from the observed SST forcing. J. Climate, 14, 77-97, doi:10.1175/1520-0442(2001)014<0077: POSCIA $>2.0 . \mathrm{CO} ; 2$.

Watkins, D. S., and C. D. Meyer, 2001: Matrix Analysis and Applied Linear Algebra. Society for Industrial and Applied Mathematics, $718 \mathrm{pp}$.

Weare, B. C., and J. S. Nasstrom, 1982: Examples of extended empirical orthogonal function analyses. Mon. Wea. Rev., 110, 481485, doi:10.1175/1520-0493(1982)110<0481:EOEEOF>2.0.CO;2.

Wilks, D. S., 2011: Statistical Methods in the Atmospheric Sciences. 3rd ed. Elsevier, 676 pp.

Wunsch, C., 2005: The total meridional heat flux and its oceanic and atmospheric partition. J. Climate, 18, 4374-4380, doi:10.1175/JCLI3539.1.

Zwiers, F. W., 1993: Simulation of the Asian summer monsoon with the CCC GCM-1. J. Climate, 6, 469-486, doi:10.1175/ 1520-0442(1993)006<0469:SOTASM $>2.0 . \mathrm{CO} ; 2$. 